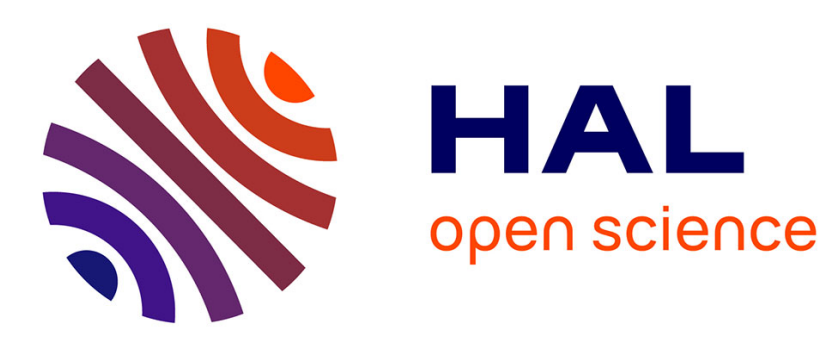

\title{
Effects of self, induced and cross phase modulations on the generation of picosecond and femtosecond white light supercontinua
}

\author{
Patrice L. Baldeck, P.P. Ho, R.R. Alfano
}

\section{To cite this version:}

Patrice L. Baldeck, P.P. Ho, R.R. Alfano. Effects of self, induced and cross phase modulations on the generation of picosecond and femtosecond white light supercontinua. Revue de Physique Appliquée, 1987, 22 (12), pp.1677-1694. 10.1051/rphysap:0198700220120167700 . jpa-00245728

HAL Id: jpa-00245728

https://hal.science/jpa-00245728

Submitted on 1 Jan 1987

HAL is a multi-disciplinary open access archive for the deposit and dissemination of scientific research documents, whether they are published or not. The documents may come from teaching and research institutions in France or abroad, or from public or private research centers.
L'archive ouverte pluridisciplinaire HAL, est destinée au dépôt et à la diffusion de documents scientifiques de niveau recherche, publiés ou non, émanant des établissements d'enseignement et de recherche français ou étrangers, des laboratoires publics ou privés. 


\title{
Effects of self, induced and cross phase modulations on the generation of picosecond and femtosecond white light supercontinua
}

\author{
P. L. Baldeck, P. P. Ho and R. R. Alfano \\ Institute for Ultrafast Spectroscopy and Lasers, and Photonics Application Laboratory, Departments of \\ Electrical Engineering and Physics, The City College of The City University of New York, New York, \\ N.Y. 10031, U.S.A.
}

(Reçu le 22 juin 1987, révisé le 16 août 1987, accepté le 24 août 1987)

\begin{abstract}
Résumé. - Les processus de génération d'impulsions lasers femtosecondes et picosecondes à très large bande spectrale sont passés en revue. Les effets des modulations de phase : automodulation (SPM), modulation induite (IPM), et modulation croisée (XPM) sont discutés. De nouvelles mesures des modulations de phase induites et croisées sont reportées.
\end{abstract}

\begin{abstract}
The effects of self, induced, and cross phase modulations on the generation of picosecond and femtosecond «white» laser pulses are reviewed. Recent measurements of cross and induced phase modulations are reported using femtosecond and picosecond laser pulses.
\end{abstract}

\section{Introduction.}

Supercontinuum generation, the generation of intense, ultrafast, and broad-spectral-width pulses, is the basis for a white-light laser source needed for numerous applications [1-4]. An alternative coherent light source to the free electron laser, the supercontinuum laser source can be wavelength selected and coded simultaneously over wide spectral ranges (up to $10000 \mathrm{~cm}^{-1}$ ) in the ultra-violet, visible and infrared regions at high repetition rates, gigawatt output peak powers, and femtosecond pulse durations. Ultrafast supercontinuum pulses have been used for time-resolved absorption spectroscopy [5-6] and material characterization [7]. Supercontinuum generation is a key step for a pulse compression technique which is used to produce the shortest optical pulses [8-9]. Future applications include signal processing, 3-D imaging, ranging, atmospheric remote sensing, and medical diagnosis $[1,3]$.

The first study on the mechanism and generation of ultrafast supercontinuum dates back to the years 1969 to 1972 when Alfano and Shapiro observed the first « white » picosecond pulse continuum in liquids and solids [10]. Spectra extended over $\sim 6000 \mathrm{~cm}^{-1}$ in the visible and IR wavelength region. They attributed the large spectral broadening of ultrafast pulses to self phase modulation (SPM) [11-14] arising from an electronic mechanism, and laid down the formulation of the supercontinuum generation model $[10,15-16]$. Over the years, the improvement of mode-locked lasers led to the production of wider supercontinua in the visible, ultra-violet and infrared wavelength regions using various materials [17-32]. A brief historical background of continuum generation is displayed in figure 1 . Recently, researchers at the Institute for Ultrafast Spectroscopy and Lasers (IUSL) of City College of New York observed and investigated induced phase modulation (IPM) and cross phase modulation (XPM) effects in optical fibers, bulk glasses and liquids [33-38].

This paper reviews our latest work on supercontinuum generation in terms of self, cross, and induced phase modulations. The first section discusses the theory of self phase modulation and presents recent measurements of supercontinuum pulse properties. New spectral effects arising from cross phase modulation and induced phase modulation are presented in the second and third sections of this paper, respectively. XPM and IPM occur when laser pulses of different wavelengths propagate simultaneously and interact through the nonlinear susceptibility coefficient $\chi^{(3)}[35,80]$. The coupled interaction leads to temporal and spectral effects induced by one pulse on the other. XPM and IPM effects are comparable to the optical Kerr effect which was first 


\begin{tabular}{|c|c|c|c|c|c|c|}
\hline Investigator & Year & Material & $\begin{array}{c}\text { Wavelength } \\
\text { Laser/Pulsewidth }\end{array}$ & Spectral & $\begin{array}{l}\text { Freq. } \\
\text { Shift }\end{array}$ & Process \\
\hline Alfano/Shapiro & $1969-1973$ & $\begin{array}{l}\text { Liquids } \\
\& \text { Solids }\end{array}$ & $\begin{array}{c}530 \mathrm{~nm} / 8 \mathrm{ps} \\
\text { or } 1060 \mathrm{~nm} / 8 \text { ps }\end{array}$ & $\begin{array}{l}\text { visible } \\
\text { near IR }\end{array}$ & $10000 \mathrm{~cm}^{-1}$ & SPM \\
\hline Stolen & $1974-1976$ & Fibers & $530 \mathrm{~nm} / \mathrm{ns}$ & visible & $500 \mathrm{~cm}^{-1}$ & SPM \\
\hline Shank/Fork et al. & 1983 & Glycerol & $620 \mathrm{~nm} / 100 \mathrm{fs}$ & $\begin{array}{l}\text { uv, visible } \\
\text { near IR }\end{array}$ & $10000 \mathrm{~cm}^{-1}$ & SPM \\
\hline $\begin{array}{l}\text { Corkum } / \mathrm{Ho} / \\
\text { Alfano }\end{array}$ & 1985 & $\begin{array}{l}\text { Semiconductor } \\
\text { Dielectrics }\end{array}$ & $10 \mu / 6 \mathrm{ps}$ & IR & $1000 \mathrm{~cm}^{-1}$ & SPM \\
\hline Corkum, Sorokin & 1986 & Gases & $\begin{array}{c}600 \mathrm{~nm} / 2 \mathrm{ps} \\
300 \mathrm{~nm} / 0.5 \mathrm{ps}\end{array}$ & $\begin{array}{l}\text { visible } \\
\text { uv }\end{array}$ & $5000 \mathrm{~cm}^{-1}$ & SPM \\
\hline $\begin{array}{l}\text { Alfano/Ho/ } \\
\text { Manassah/Jimbo }\end{array}$ & 1986 & Glass & $\begin{array}{c}1060 \mathrm{~nm} / 530 \mathrm{~nm} \\
8 \mathrm{ps}\end{array}$ & visible & $1000 \mathrm{~cm}^{-1}$ & IPM \\
\hline $\begin{array}{l}\text { Alfano/Ho/ } \\
\text { Wang/Jimbo }\end{array}$ & 1986 & $\mathrm{ZnSe}$ & $\begin{array}{c}1060 \mathrm{~nm} \\
8 \mathrm{ps}\end{array}$ & visible & $1000 \mathrm{~cm}^{-1}$ & ISB \\
\hline $\begin{array}{l}\text { Alfano/Ho/ } \\
\text { Baldeck }\end{array}$ & 1987 & Fibers & $\begin{array}{c}530 \mathrm{~nm} \\
30 \mathrm{ps}\end{array}$ & visible & $1000 \mathrm{~cm}^{-1}$ & XPM \\
\hline
\end{tabular}

Fig. 1. - Historical table of continuum generation with ultrafast laser sources.

observed in the early 1970's [83-84]. XPM describes phase modulations which are introduced by pump pulses on weaker pulses generated from the noise (stimulated Raman scattering, four photon parametric generation, ...). XPM is intrinsic to the generation process and is not controlled by the experimentator. IPM corresponds to phase modulations which are induced on a probe pulse by the experimentator using a command pulse.

\section{Self phase modulation (SPM).}

Nonlinear optical phenomena such as self phase modulation, stimulated Raman scattering, four photon parametric generation, harmonic generation, and three wave mixing are responsible for spectral broadening and frequency generation [39-41, 50]. Self phase modulation is one of the main processes responsible for supercontinuum generation by picosecond and femtosecond pulses. In this section, we review the SPM theory, present measurements of spectral and temporal SPM effects, and discuss the importance of self-focusing, group velocity dispersion, self steepening and initial pulse chirping on the supercontinuum generation.

\subsection{SELF PHASE MODULATION THEORY.}

2.1.1 Nonlinear and dispersive wave equation. The optical electromagnetic field of supercontinuum pulses mut ultimately satisfy Maxwell's wave vector equation

$$
\nabla \times(\nabla \times \mathbf{E})+1 / c^{2} \partial^{2} \mathbf{E} / \partial t^{2}=-\mu_{0} \partial^{2} \mathbf{P} / \partial t^{2}
$$

which can be reduced to [42]

$$
\begin{aligned}
& i\left(\partial A / \partial z+k^{(1)} \partial A / \partial t\right)-1 / 2 k^{(2)} \partial^{2} A / \partial t^{2}- \\
& -i / 6 k^{(3)} \partial^{3} A / \partial t^{3}+i \gamma A+\bar{n}_{2} k_{0}|A|^{2} A \\
& \quad+i 2 n_{2} / c \partial\left(|A|^{2} A\right) / \partial t+i r A \partial|A|^{2} / \partial t=0
\end{aligned}
$$

where $A(z, t)$ is the complex envelope of the electric field, $k^{(n)}=\partial^{n} k / \partial \omega^{n}$ are evaluated at $\omega_{0}$, the total refractive index $n$ is defined by $n^{2}=\chi^{(1)}+$ $3 / 4 \chi^{(3)} \cong n_{0}^{2}+2 n_{0} n_{2}|A(t)|^{2}, \gamma$ is for the medium losses, $\bar{n}_{2}=1 / 2 n_{2}$ is the effective nonlinear refractive index, and the last term has been added to take into account the time retardation effect in $\chi^{(3)}$.

Equation (2) is the third-order nonlinear and dispersive equation which has been found to describe the characteristics of femtosecond pulses propagating in optical fibers [43]. Equation (2) has been derived using the following approximations: 1) linearlypolarized electric fields, 2) homogeneous radial fields, 3) slowly-varying envelope, 4) isotropic and non magnetic medium, 5) negligible Raman effect, and 6) wavelength-independent nonlinear susceptibility $\chi^{(3)}$.

In equation (2), the first two terms describe the envelope propagation at the group velocity $v_{\mathrm{g}}=$ $\left[k^{(1)}\right]^{-1}$. The 3rd and 4th terms determine the temporal pulse broadening due to the group velocity dispersion. The 5 th term is for the medium losses while the 6th term characterizes the first order of the nonlinear polarization, which is responsible for the self phase modulation effect and spectral broadening. The 7 th term describes the pulse steepening which occurs because the pulse peak propagates slower due to the nonlinear refractive index $n_{2} A^{2}$. 
Finally, the 8th term leads to the self-frequency shift [44-45]. In supercontinuum generation, the temporal and spectral pulse shapes (extent, asymmetry, and modulation structure) are affected by the above terms which occur simultaneously and interactively. However, equation (2) does not describe completely supercontinuum processes for it does not take into account phenomena such as self-focusing, four-photon parametric generation, and stimulated Raman scattering. One needs the fastest computers to solve this problem completely.

2.1.2 Self phase modulation mechanism [16]. - The basic mechanism and properties of SPM can be explored using the simplified nonlinear wave equation :

$$
\partial A / \partial z+1 / v_{\mathrm{g}} \partial A / \partial t=i\left(\omega_{0} / 2 c\right) n_{2}|A|^{2} A
$$

which is obtained from equation (2) after neglecting group velocity dispersion, absorption, self-steepening and self-frequency shift.

Denoting $a$ and $\alpha$ the amplitude and phase of the electric field envelope $A=a \mathrm{e}^{i \alpha}$, equation (3) reduces to

$$
\begin{gathered}
\partial a / \partial z+1 / v_{\mathrm{g}} \partial a / \partial t=0 \\
\partial \alpha / \partial z+1 / v_{\mathrm{g}} \partial \alpha / \partial t=\left(\omega_{0} / 2 c\right) n_{2} a^{2} .
\end{gathered}
$$

The analytical solutions are

$$
a(\tau)=a_{0} F(\tau)
$$

and

$$
\begin{aligned}
\alpha(z, \tau)=\left(\omega_{0} / 2 c\right) & n_{2} \int_{0}^{z} a^{2} \mathrm{~d} z^{\prime}= \\
& =\left(\omega_{0} / 2 c\right) n_{2} a_{0}^{2} F^{2}(\tau) z
\end{aligned}
$$

where $F(\tau)$ is the pulse shape envelope and $\tau$ is the local time $\tau=t-z / v_{\mathrm{g}}$.

The electric field envelope solution of equation (3) is given by

$$
A(z, \tau)=a(\tau) \exp \left[i\left(\omega_{0} / 2 c\right) n_{2} a_{0}^{2} F^{2}(\tau) z\right] .
$$

The main physics of the supercontinuum generation is contained in equation (6). As shown in figure $2 a$, the phase of a pulse propagating in a distorted medium becomes time-dependent. There is self phase modulation, and the electric field frequency is shifted (Fig. 2b).

Since the pulse duration is much larger than the optical period $2 \pi / \omega_{0}$ (slowly-varying approximation), the electric field at each position $\tau$ within the pulse shape has a specific local and instantaneous frequency which is given by

$$
\omega(\tau)=\omega_{0}+\delta \omega(\tau)
$$
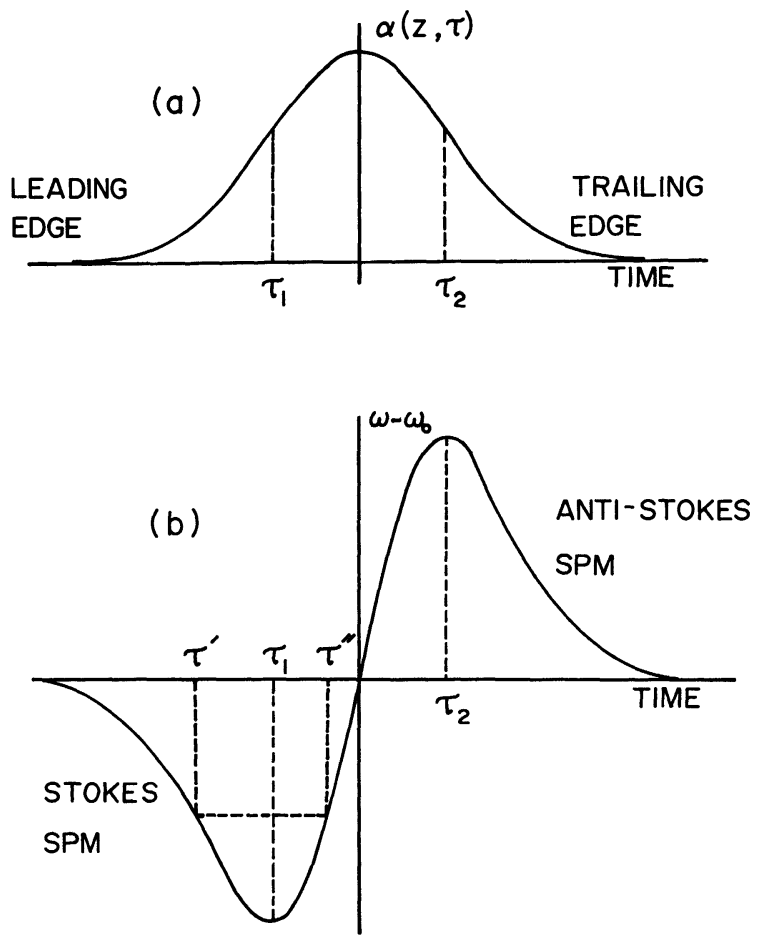

Fig. 2. - (a) Time-dependent phase $\alpha(z, t)$ generated by self phase modulation (SPM). (b) Time distribution of SPM-shifted frequencies $\partial \omega(z, t)=-\partial \alpha / \partial t$.

where

$$
\begin{aligned}
\delta \omega(\tau)=-\partial \alpha / \partial \tau & = \\
& =-\left(\omega_{0} / 2 c\right) n_{2} a_{0}^{2} z \partial F^{2} / \partial \tau .
\end{aligned}
$$

The $\delta \omega(\tau)$ is the frequency shift generated at the time location $\tau$ of the pulse shape. The frequency shift is proportional to the derivative of the pulse envelope. Thus, the frequency shift varies at each point of the pulse shape. It corresponds to the generation of new frequencies which result in wider spectra, and shorter pulses at given frequencies. Figure $2 b$ shows the frequency distribution of the electric field within the pulse shape. The leading edge, the pulse peak, and the trailing edge have redshifted, non-shifted and blue-shifted frequencies, respectively. The maximum frequency broadening on Stokes and anti-Stokes sides occurs at the inflection points $\tau_{1}$ and $\tau_{2}$. Moreover, identical frequency shifts appear at different time locations. For example, in figure $2 b$ the points $\tau^{\prime}$ and $\tau^{\prime \prime}$ have the same frequency shift $\delta \omega$ and the same absolute frequency $\omega=\omega_{0}+\delta \omega$. The electric field amplitude spectrum is obtained by taking the Fourier transform of the complex temporal envelope $A(z, \tau)$ :

$$
A(\Omega, z)=1 / 2 \pi \int_{-\infty}^{+\infty} A(z, \tau) \exp [i \Omega \tau] \mathrm{d} \tau
$$

where $\Omega=\omega-\omega_{0}$. 
The intensity spectrum is given by

$$
S(\Omega, z)=(c / 4 \pi)|A(\Omega, z)|^{2} .
$$

In practical cases, the phase of $A(z, \tau)$ is large compared with $\pi$, and the method of stationary phase [46-47] leads to

$$
\Delta \omega(z)_{\max }=\left(\omega_{0} / 2 c\right) n_{2} a_{0}^{2}\left[\partial F^{2} /\left.\partial \tau\right|_{\tau_{1}}-\partial F^{2} /\left.\partial \tau\right|_{\tau_{2}}\right] z
$$

and,

$$
\begin{aligned}
S(\Omega, z)=(c / 4 \pi)\left(4 \pi c / \omega_{0} n_{2} z\right) & \left\{F\left(\tau^{\prime}\right)^{2} /\left[\partial^{2} F^{2} /\left.\partial \tau^{2}\right|_{\tau^{\prime}}\right]+F\left(\tau^{\prime \prime}\right)^{2} /\left[\partial^{2} F^{2} /\left.\partial \tau^{2}\right|_{\tau^{\prime \prime}}\right]+\right. \\
+ & 2\left[F\left(\tau^{\prime}\right) F\left(\tau^{\prime \prime}\right)\right] /\left[\partial^{2} F^{2} /\left.\partial \tau^{2}\right|_{\tau^{\prime}} \partial^{2} F^{2} /\left.\partial \tau^{2}\right|_{\tau^{\prime \prime}}\right]^{0.5} \times \cos \left[\Omega\left(\tau^{\prime}-\tau^{\prime \prime}\right)\right. \\
+ & \left.\left.\omega_{0} / 2 c n_{2} a_{0}^{2} z\left(F\left(\tau^{\prime}\right)^{2}-F\left(\tau^{\prime \prime}\right)^{2}\right)\right]\right\}
\end{aligned}
$$

where $\Delta \omega_{\max }$ is the maximum frequency spread, $\tau_{1}$ and $\tau_{2}$ are the pulse envelope inflection points, and $\tau^{\prime}$ and $\tau^{\prime \prime}$ are the points of the pulse shape which have the same frequency $\omega=\omega_{0}+\delta \omega$.

The last term of equation (11) produces the periodic structure in the SPM spectrum via interferences of the frequency phases generated at the points $\tau^{\prime}$ and $\tau^{\prime \prime}$. Notice that the spectral structures cannot be explained by theories based on the linear chirp approximation for which a specific frequency shift corresponds to an unique time $\tau$.

An estimate of the modulation frequency $\delta \omega_{M}$ can be made by calculating the maximum number of interference minima, and dividing this number into the maximum frequency broadening. A straightforward calculation leads to

$$
\begin{aligned}
\delta \omega_{M} \approx 2 \pi \partial F^{2} /\left.\partial \tau\right|_{\tau_{1}} / F\left(\tau_{1}\right)^{2} & = \\
& \approx 2 \pi \partial F^{2} /\left.\partial \tau\right|_{\tau_{2}} / F\left(\tau_{2}\right)^{2} .
\end{aligned}
$$

Assuming Gaussian shaped pulses $F(\tau)=$ $\exp \left[-\tau^{2} / 2 \tau_{0}^{2}\right]$ the maximum frequency broadening $\Delta \omega(z)_{\max }$ is given by $[16,48,49]$

$$
\Delta \omega(z)_{\max } \sim\left(\omega_{0} / c\right) n_{2} a_{0}^{2} z / \Delta \tau_{0} .
$$

The total spectral width $\Delta \omega(z)$ is given by

$$
\Delta \omega(z)^{2}=\Delta \omega_{0}^{2}+\Delta \omega_{\max }(z)^{2} .
$$

The modulation period $\delta \omega_{M}$ by

$$
\delta \omega_{M}=4 \pi / \Delta \tau_{0}
$$

where $\Delta \omega_{0}$ and $\Delta \tau_{0}$ are the initial spectral and temporal pulses ${ }^{*}$ widths, respectively. From equation (13a) the spectral broadening is proportional to the pulse peak power, the interaction length, and inversely proportional to the pulse duration. However, the average modulation period depends only on the inverse of the pulse duration.

The chirping - the temporal distribution of frequencies in the pulse shape or frequency sweep is an important characteristic of SPM broadened pulses. In the linear chirp approximation, the chirp coefficient $c$ is usually defined by the phase relation

$$
\alpha=c \tau^{2} .
$$

For a Gaussian electric field envelope and linear approximation, the envelope reduces to

$$
F^{2}(\tau)=\exp \left[-\tau^{2} / \tau_{0}^{2}\right] \approx 1-\tau^{2} / \tau_{0}^{2}
$$

The linear chirp coefficient derived from equations (6) and (15) becomes

$$
c=\left(\omega_{0} / 2 c\right) n_{2}\left(a_{0}^{2} / \tau_{0}^{2} z\right) .
$$

The above analytical expressions for the spectral broaclening, spectral modulation period, and linear chirp coefficients have been derived from the simplest form of the nonlinear wave equation which does not include the effects of group velocity dispersion and self steepening.

2.2 MEASUREMENTS OF SELF PHASE MODULATION. - The first ultrafast supercontinuum pulses were generated by passing intense picosecond pulses into several centimeters of transparent liquids and solids [10]. The spectral broadening was attributed to SPM arising from the nonlinear response of media, primarily from electronic cloud distortion. The measurements showed that spectra were much wider than predicted by the above theory using the pulse duration. The sub-structure of about $0.1 \mathrm{ps}$ within the picosecond pulse emitted by a mode locked glass laser was needed to explain the broadening due to SPM.

In the following, we review the experimental evidences of SPM.

2.2.1 Experimental arrangement. - Spectral measurements have been performed using the output from a CPM ring dye laser combined with a fourstage YAG-pumped dye amplifier system. Pulses of 500 femtosecond duration at $625 \mathrm{~nm}$ were amplified to an energy of about $1 \mathrm{~mJ}$ at a repetition rate of 
$20 \mathrm{~Hz}$. Pulses were weakly focused into the sample. Output pulses were imaged on the slit of a $1 / 2 \mathrm{~m}$ Jarell-Ash spectrometer and spectra were recorded using an optical multichannel analyser OMA2.

The spectral and temporal shapes of SPM broadened picosecond pulses have also been studied using pulses of 8 and 25 picosecond duration. These pulses were generated at 527 and $532 \mathrm{~nm}$ by frequency-doubled mode-locked $\mathrm{Nd}$ : glass and modelocked Nd: YAG lasers, respectively. Temporal shapes were measured with a 2 ps-resolution Hamamatsu streak camera.
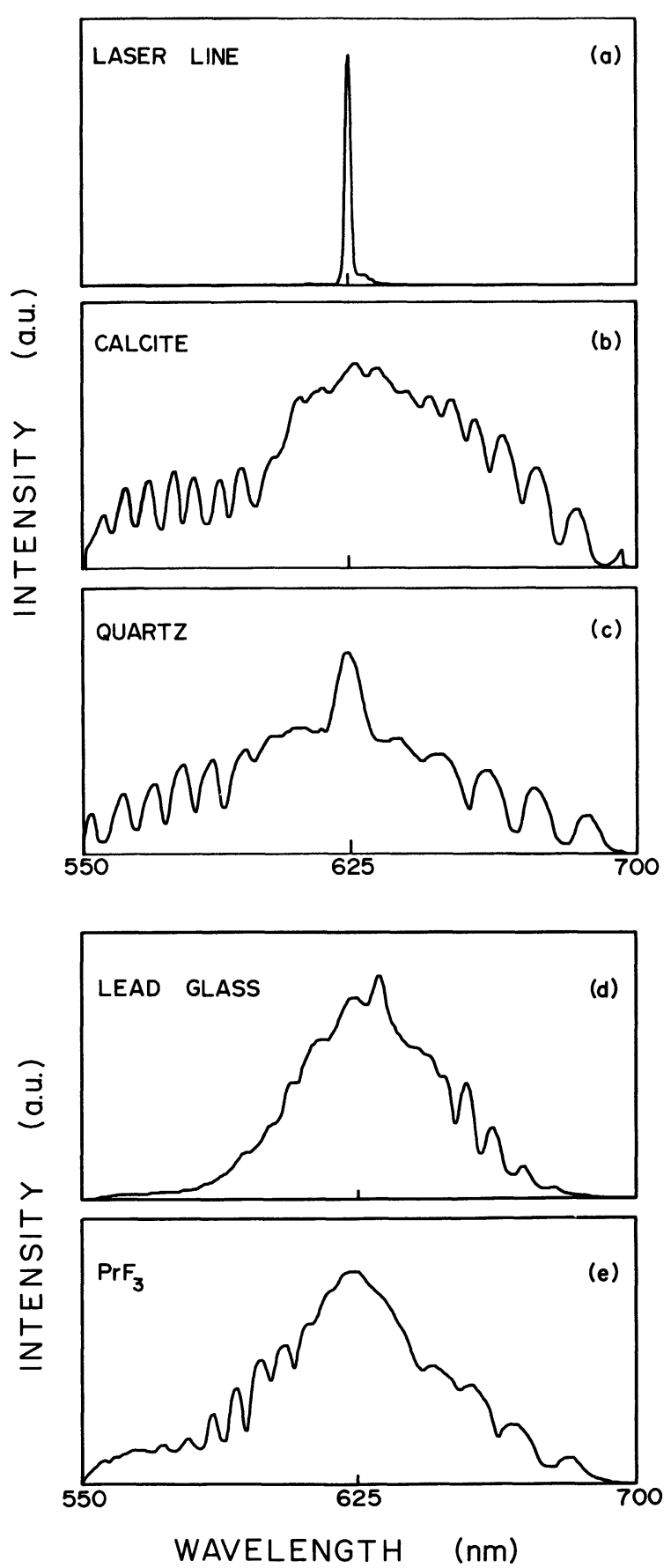

In the present work, femtosecond continua were generated in various solids and liquids such as caicite, quartz, water, benzene, ethanol, lead glass, pyrex, $\mathrm{PrF}_{3}, \mathrm{KNiF}_{3}$ and silica optical fibers. Sample thicknesses ranged in the $2-10 \mathrm{~mm}$ range. A singlemode optical fiber at visible wavelengths was custom-made by Corning Glass. The cut-off wavelength was $462 \mathrm{~nm}$, the core diameter $2.5 \mu \mathrm{m}$, and the refractive index difference $0.24 \%$.

2.2.2 General experimental observations. - Typical supercontinuum spectra generated by 500 fs pulses
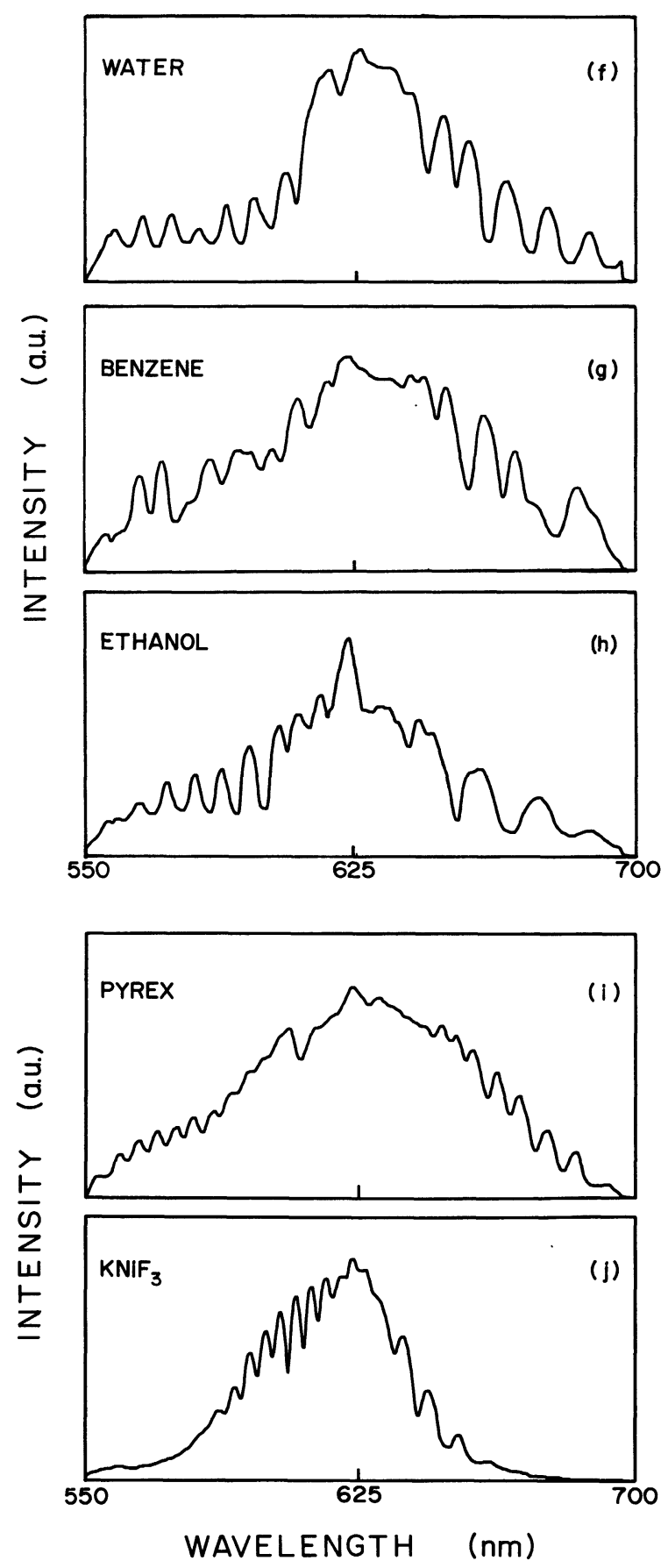

Fig. 3. - Spectra of femtosecond supercontinuum pulses in various media. 
are displayed in figure 3. For reference, the laser spectrum is shown in figure 3a. All continuum spectra were similar despite the different materials. The spectra were wide and modulated. Spectral shapes, extents and modulation periods changed significantly from shot to shot using the same material. Spectra seemed to depend more on pulse fluctuations (time duration and peak power) than on the state of the material : solids (dielectric, semiconductor, magnetic) or liquids. Spectral widths extended up to $5000 \mathrm{~cm}^{-1}$. Most of the laser energy was frequency broadened and there was no well defined intense spike at the laser frequency. Spectral modulation periods ranged from 100 to $350 \mathrm{~cm}^{-1}$ with an average of about $160 \mathrm{~cm}^{-1}$.

2.2.3 Maximum spectral extent. - The simplified SPM theory predicts a SPM spectral extent varying as $\Delta \omega(z)_{\max } \approx\left(\omega_{0} / c\right) n_{2} a_{0}^{2} z / \Delta \tau_{0}$. The maximum SPM spectral width has been investigated as func-

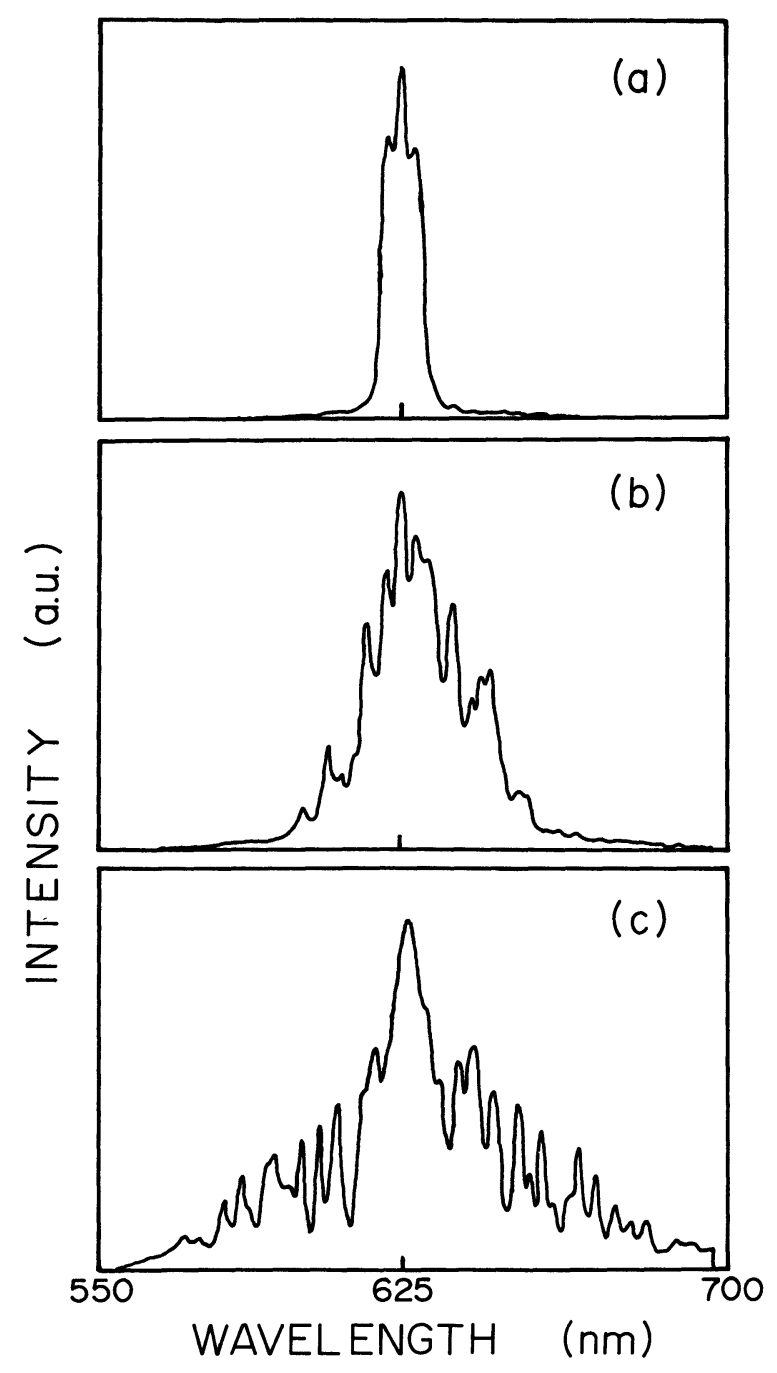

Fig. 4. - Sequence of spectral broadening versus increasing input energy in a singlemode optical fiber (length $=$ $30 \mathrm{~cm}$ ). The pump intensity was increased from a) to c). tions of the input-pulse peak power $a_{0}^{2}$, and sample length $z$. The experiment was performed using a singlemode optical fiber for which the peak power and interaction length could be better controlled than for bulk materials. In bulk materials, intense laser beams self-focus and break into instable filaments. Figure 4 shows a typical sequence of spectral broadening versus increasing input peak powers using $500 \mathrm{fs}$ pulses. As for the other samples, the spectra were modulated. The spectral extent is plotted against input pulse energy in figure 5. The relative energy of each pulse was calculated by integrating its total broadened spectral distribution. As predicted, the supercontinuum extent increased linearly with the input pulse intensity. The length dependence of spectral broadening is plotted in figure 6. The broadening was independent of the optical fiber length. This arised because of pulse

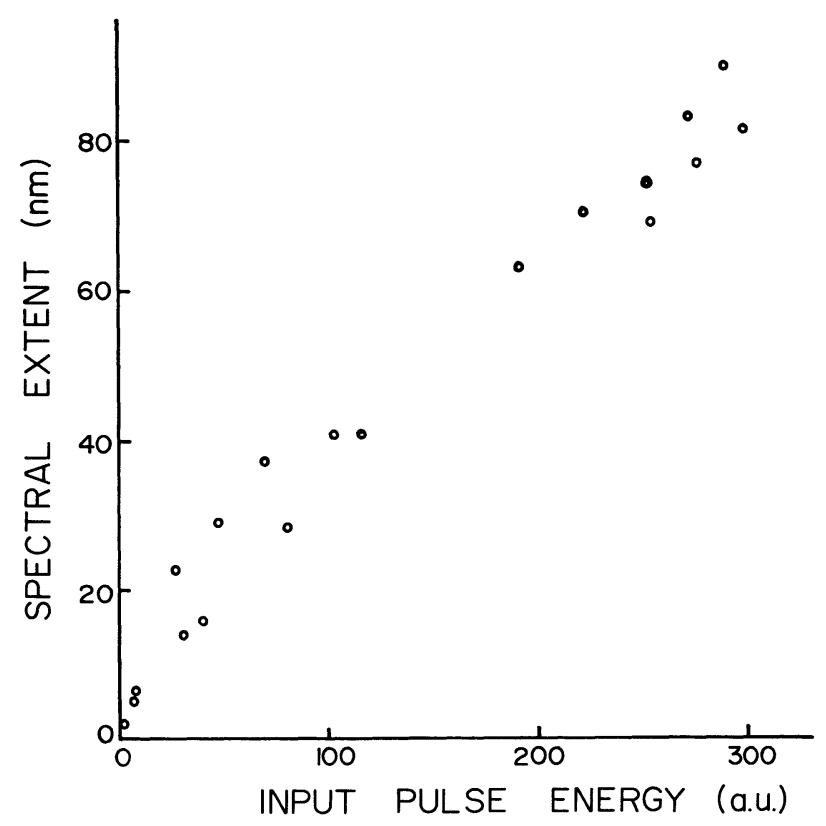

Fig. 5. - SPM spectral broadening versus input pulse energy in a singlemode optical fiber (length $=30 \mathrm{~cm}$ ).

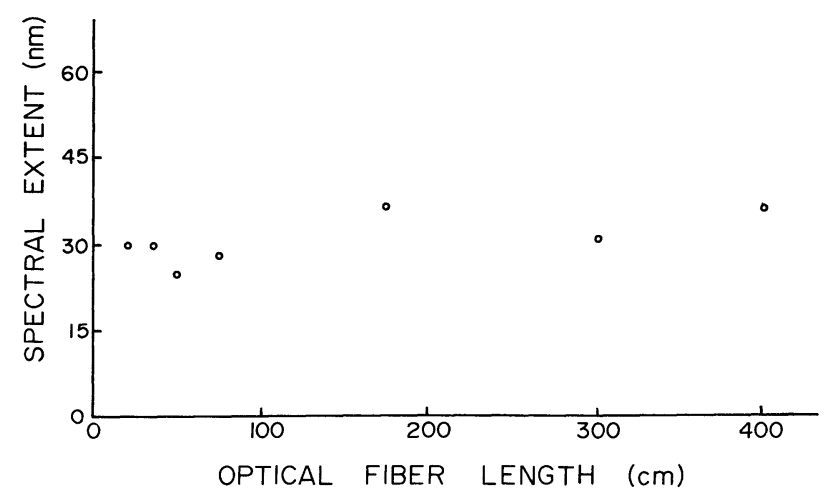

Fig. 6. - SPM spectral broadening versus optical fiber length. 
broadening due to group velocity dispersion. The SPM spectral-broadening occurred in the first few centimeters of fiber for femtosecond pulses.

2.2.4 Spectral modulation. - As shown in figure 3 the spectral modulation is a definite characteristic of supercontinuum spectra. The spectral modulation period can be estimated using $\delta \omega_{0}=4 \pi / \Delta \tau_{0}$. Using this relation, the average modulation period of $160 \mathrm{~cm}^{-1}$ corresponds to an initial pulse duration of $400 \mathrm{fs}$ which is in good agreement with the $500 \pm 100$ fs pulse duration of the CPM laser and the bandwidth of incident pulses.

SPM spectra measured with the Nd: YAG laser had a $40-\mathrm{cm}^{-1}$ modulation which corresponds to a pulse duration of $5 \mathrm{ps}$. This was shorter than the 25 ps-pulse duration but was close to the coherence pulse duration and fine substructure of about 2 ps.

One should notice that spectral modulations were not always pronounced as shown in figure 7 . These figures represent the anti-Stokes part of supercontinuum spectra generated by weakly focusing 500 -fs time duration pulses into a $20 \mathrm{~cm}$-long cell filled with ethanol. From right to left one sees the broadened pump, a distribution of four-photon parametric frequencies and the SPM and XPM broadened antiStokes Raman. Spectra of figures $7 \mathrm{a}$ and $7 \mathrm{~b}$ were measured under the same experimental conditions for two successive laser shots. In figure $7 \mathrm{a}$ there is slight modulation; however, in figure $7 \mathrm{~b}$ the modulation is very regular. The supercontinua were generated in self-focused filaments due to the long cell length. For the spectum of figure $7 \mathrm{~b}$ the filament could have been focused on the spectrometer slit, while for figure $7 \mathrm{a}$ the filament could have been out of focus.

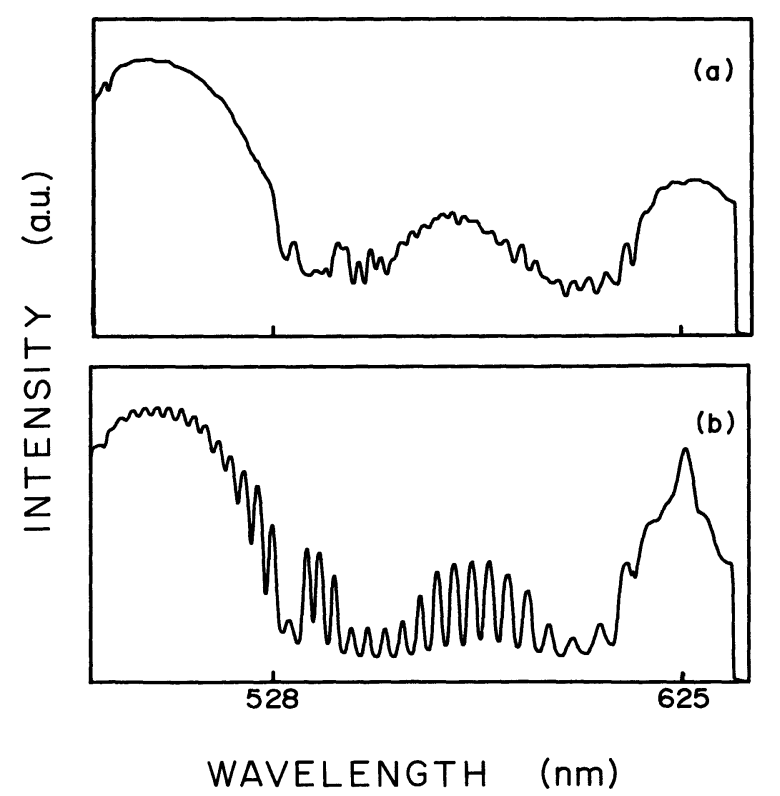

Fig. 7. - Anti-Stokes spectra of supercontinua generated in a $20 \mathrm{~cm}$-long cell filled with Ethanol. a) and b) correspond to two successive laser shots.
2.2.5 Chirp-frequency sweep. - The chirp (frequency sweep) of supercontinuum pulses is one of the major signature of SPM broadened pulses [23, 51]. It has become an important characteristic of SPM broadened pulses used for pulse compression using optical fibers. The frequency sweep of supercontinuum pulses generated by 8 ps pulses propagating in $\mathrm{CCl}_{4}$ is shown in figure 8. The chirp has been

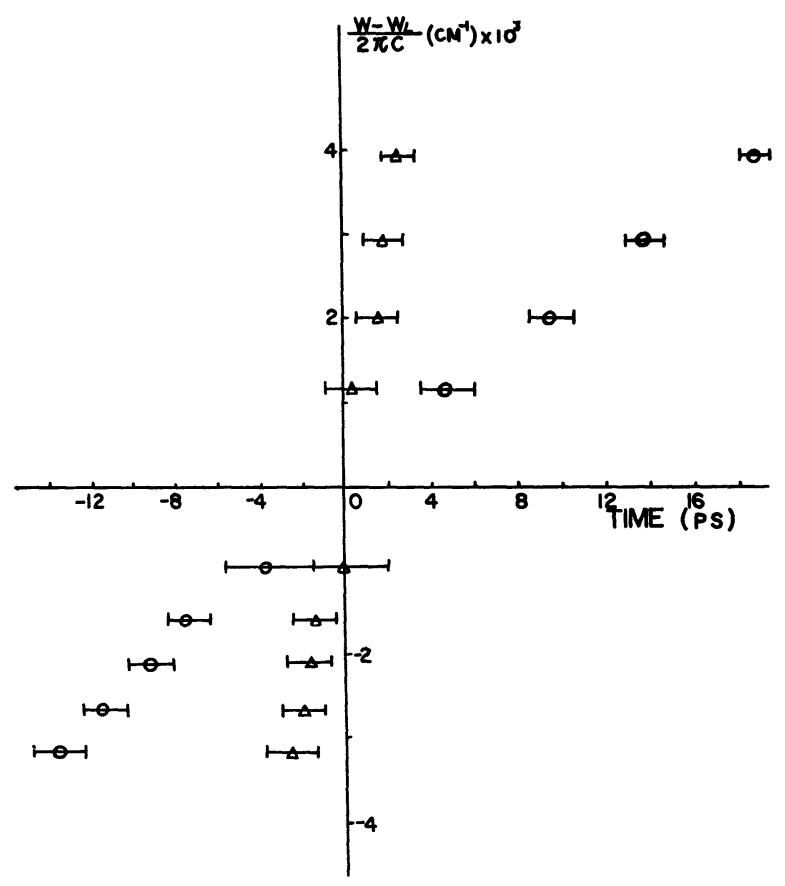

Fig. 8. - Measured supercontinuum temporal distribution at different wavelengths : $O$, data points with correction of the optical path in filters; $\Delta$, data points with the correction of both the optical path in filters and group velocity dispersion in liquid [52].

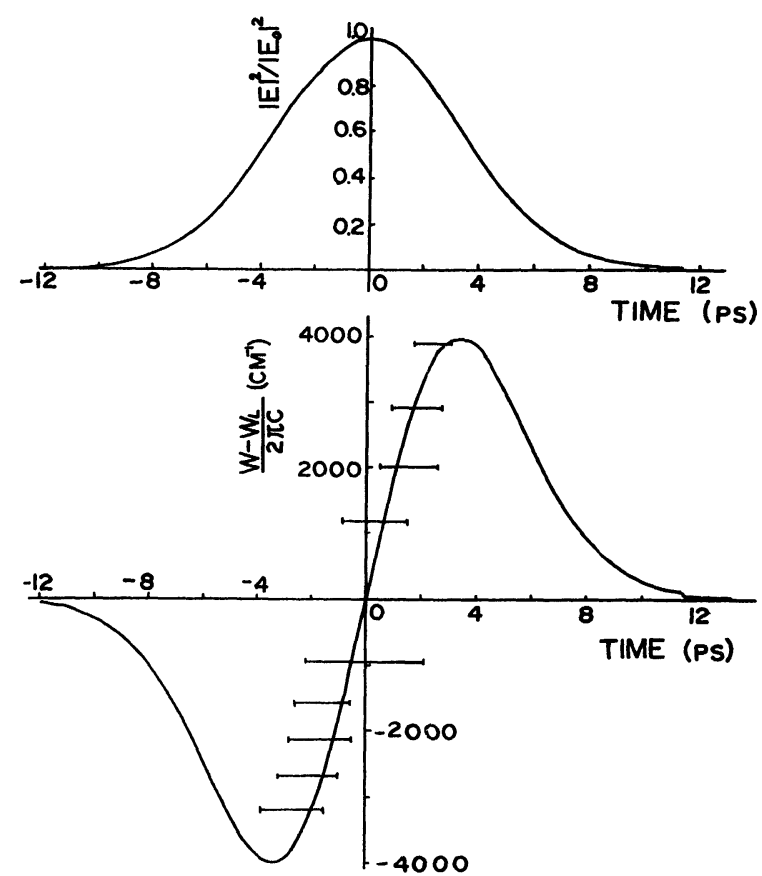

Fig. 9. - Comparison of the measured temporal distribution of supercontinuum with the SPM model [52]. 
recently measured in our laboratory with a 2 psresolution streak camera [52]. The results are in agreement with the above theory shown in figure $2 b$ and figure 9. Recently, Dorsinville et al. [53] generated supercontinuum pulses by focusing 25 ps-timeduration pulses into a $5 \mathrm{~cm}$-long cell filled with $\mathrm{D}_{2} \mathrm{O}$. Using $10 \mathrm{~nm}$-bandwidth narrow band filters, they were able to generate tunable pulses of less than 3-ps in the range $480-590 \mathrm{~nm}$ (Fig. 10) showing that for each narrow spectral band of a SPM pulse there corresponds a narrow pulse in time.

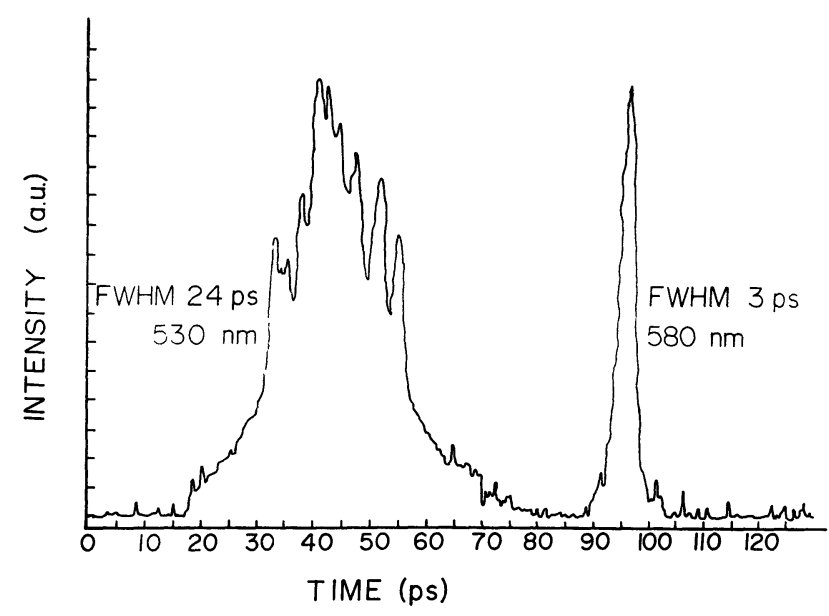

Fig. 10. - Streak camera temporal profile of the $530 \mathrm{~nm}$ incident laser pulse and $10 \mathrm{~nm}$ bandwidth pulse at $580 \mathrm{~nm}$ [53]. The 3-ps pulse was obtained by spectral filtering a SPM frequency continuum generated in $\mathrm{D}_{2} \mathrm{O}$.

2.3 HIGHER ORDER EFFECTS ON SELF PHASE MODULATION. - A complete description of SPM-generated spectral broadening should take into account higher-order effects such as self-focusing, group velocity dispersion, self-steepening, and initial pulse chirping. These effects change the observed spectral and temporal profiles.

2.3.1 Self-focusing. - In the earliest experiments using ps-pulses, supercontinuum pulses were often generated in small-scale filaments which resulted from the self-focusing of intense laser beams [10]. Self-focusing arises from the radial dependence of the nonlinear refractive index $n(r)=n_{0}+n_{2} E^{2}(r)$ [54]. It has been observed in many liquids, bulk materials, and most recently in optical fibers [55]. Its effects on the supercontinuum pulse generation can be viewed as good and bad. On one hand, it facilitates the spectral broadening by concentrating the laser beam energy. On the other hand, selffocusing is a random and unstable phenomenon which is not as controllable as one would prefer for the design of a «white light laser source ». However, femtosecond supercontinua are generated with thinner samples that picosecond supercontinua which reduces, but does not totally eliminate self-focusing effects.
2.3.2 Group velocity dispersion. - Group velocity dispersion (GVD) arises from the wavelength dependence of the group velocity. The first order GVD term $k^{(2)}$ leads to a symmetrical temporal broadening [56-59]. A typical value for the broadening rate arising from $k^{(2)}$ is $500 \mathrm{fs} / \mathrm{m} . \mathrm{nm}$ (in silica at $532 \mathrm{~nm}$ ). In the case of supercontinuum generation, spectral widths are generally large (several hundred of $\mathrm{nm}$ ), but interaction lengths are usually small $(<1 \mathrm{~cm})$. Therefore, the temporal broadening arising from GVD is often small for picosecond pulses but is important for femtosecond pulses. Limitations on spectral extents of supercontinuum generation are also related to GVD. Although the spectral broadening should increase linearily with the medium length, i.e. $\Delta \omega(z)_{\max } \approx\left(\omega_{0} / c\right) n_{2} a^{2} z / \Delta \tau$, it quickly reaches a maximum as shown in figure 6 . This is because GVD, which is large for pulses having SPMbroadened spectra, reduces the pulse peak power $a^{2}$ and broadens the pulse duration $\Delta \tau$. As shown in figure 8 , the linear chirp parameter is decreased by the GVD chirp in the normal dispersion regime. This effect is used to linearize chirps in the pulse compression technique.

The second-order term $k^{(3)}$ has been found responsible for asymmetrical distortion of temporal shapes and modulation of pulses propagating in the lowest region of silica optical fibers [60]. Since the spectra of supercontinuum pulses are exceptionally broad, this term should also lead to asymmetrical distortions of temporal and spectral shapes of supercontinuum pulses generated in thick samples.

2.3.3 Self-steepening. - Pulse shapes and spectra of intense supercontinuum pulses have been found to be asymmetric [61]. There are two potential sources for asymmetrical broadening in supercontinuum generation. The first one arises from the second order GVD term $k^{(3)}$. The second one is selfsteepening, which is intrinsic to the SPM process and occurs even in non dispersive media.

Due to the intensity and time dependence of the refractive index, $n=n_{0}+n_{2} E(t)^{2}$, the supercontinuum-pulse peak sees a higher refractive index than its edges. Because of $v=c / n$, the pulse peak travels slower than the leading and trailing edges. This results in a sharpened trailing edge. Selfsteepening occurs and more blue-shifted frequencies (sharp trailing edge) are generated than red-frequencies. Several theoretical approaches have given approximate solutions for the electric field envelope distorted by self-steepening and asymmetrical spectral extents [62-65]. Actual self steepening effects have not been clearly observed in the time domain.

2.3.4 Initial pulse chirping. - Most femtosecond and picosecond pulses are generated with initial chirps. These chirps arise mainly from GVD and 
SPM which occur in the laser cavity [66]. Recently, Agrawal has computed the nonlinear and dispersive equation for initially chirped picosecond pulses [67]. As shown in figures 11 , the spectral broadening is reduced for positive chirps and enhanced for negative chirps in the normal dispersion regime. Moreover, the spectral distribution of SPM is also affected by the initial chirp.

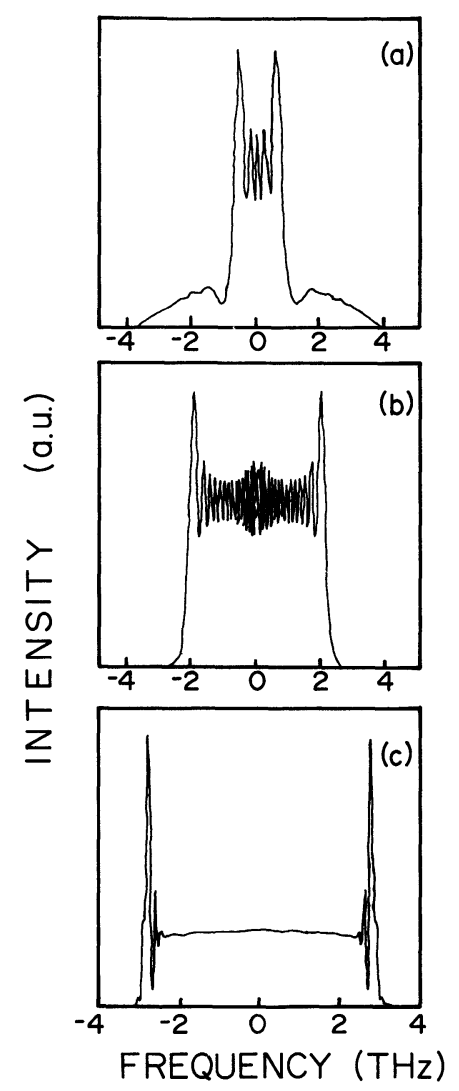

Fig. 11. - Influence of initial pulse chirping on SPMbroadened spectra in optical fibers [67]. Peak power = $1000 \mathrm{~W}$. (a) $c=50$ (b) $c=0$ (c) $c=-50$.

\section{Cross phase modulation (XPM).}

When long samples are used, stimulated Raman scattering (SRS) can contribute to the formation of ultrafast continua. In 1980, Gersten, Alfano and Belic predicted that ultrashort pulses should generate broad Raman lines due to the coupling among the laser photons and vibrations. This phenomenon was called cross phase modulation (XPM) [35]. XPM is comparable to SPM. It corresponds to the phase modulation of the Raman pulse by the intense pump laser pulse. XPM occurs while the Raman pulse is generated from noise and amplified. XPM is a phenomenon intrinsic to the Raman process. As a consequence, the pump pulse frequency is not tunable because once the frequency of the signal (weak beam) is chosen, the signal-pump frequency difference must satisfy the Raman shift characteristic of the medium. Thus, the XPM modulation is hardly controllable, which reduces its potential applications.

The XPM terminology defines the phase modulations of pulses generated from the noise at new frequencies. For example, XPM occurs in nonlinear optical processes such as stimulated Raman scattering, second harmonic generation, and stimulated four photon parametric generation. However, this terminology does not include induced phase modulation (IPM) which corresponds to phase modulations produced by input pulses of different wavelengths interacting in a nonlinear medium (see Sect. 4). The induced phase modulation is totally controlled by the experimentator at the medium entrance by chosing the interaction medium and pulse characteristics (peak powers, pulse durations, wavelengths and pulse delays).

In 1981, Cornelius and Harris stressed the role of SPM in SRS from more than one mode [68]. Lately, a great deal of attention has been focused on the combined effects of SRS, SPM and GVD for the purposes of pulse compression and soliton generation [69-75]. Schadt et al. derived and computed the coupled waves equations describing the changes of pump and Raman envelopes in nonlinear and dispersive optical fibers [76-77]. Extending the work of Gersten, Alfano and Belic, Manassah obtained analytical solutions for Raman pulse shapes in presence of pulse walk-off [78]. Very recently, our group measured and characterized the spectral effects of XPM on picosecond pulses propagating in optical fibers [36-38].

In the followings theory and the latest experimental results of XPM using picosecond pulses in optical fibers and femtosecond pulses in ethanol are highlighted.

3.1 XPM THEORY. - There are two different approaches for modeling XPM. The first one uses the computation of coupled nonlinear and dispersive wave equations describing the pump and generated Raman pulse envelopes. Recently, Schadt et al. theoretically studied the propagation of 100 -ps pulses in singlemode optical fibers [76-77]. The second approach uses the derivation of analytical solutions with some approximations. It was first developed by Gersten et al. [35] and recently extended by Manassah [78].

In the presence of co-propagating Raman and pump pulses the nonlinear polarization can be approximated (instantaneous response, etc.) by

$$
P^{(\mathrm{NL})}(z, t)=\varepsilon_{0} \chi^{(3)} E(z, t) E(z, t) E(z, t)
$$

$E(z, t)$ is the total electric field defined by

$$
\begin{gathered}
E(z, t)=1 / 2\left\{\left[A_{\mathrm{P}}(z, t) \exp \left[i\left(\beta_{\mathrm{P}} z-\omega_{\mathrm{P}} t\right)\right]\right]+\right. \\
\left.+\left[A_{\mathrm{R}}(z, t) \exp \left[i\left(\beta_{\mathrm{R}} z-\omega_{\mathrm{R}} t\right)\right]\right]+\text { c.c. }\right\}
\end{gathered}
$$


where $P$ and $R$ refer to the pump and the Raman electric fields, respectively. The anti-Stokes Raman is neglected. Substituting equation (18) into equation (17) and keeping only terms synchronous with either pump or Stokes carrier frequency, the nonlinear polarization becomes [76]

$$
\begin{aligned}
& P_{\mathrm{P}}^{(\mathrm{NL})}(z, t)=1 / 2\{[i 3 / 2 \chi_{\mathrm{r}}\left|A_{\mathrm{R}}(z, t)\right|^{2}+3 / 4 \chi_{\mathrm{pm}} \times \\
&\left.\left.\left.\times\left(\left|A_{\mathrm{P}}(z, t)\right|^{2}+2\left|A_{\mathrm{R}}(z, t)\right|^{2}\right)\right] \times A_{\mathrm{P}}(z, t) \exp \left[i\left(\beta_{\mathrm{P}} z-\omega_{\mathrm{P}} t\right)\right]\right]+ \text { c.c. }\right\}
\end{aligned}
$$

and

$$
\begin{aligned}
P_{\mathrm{R}}^{(\mathrm{NL})}(z, t)=1 / 2\left\{\left[-i 3 / 2 \chi_{\mathrm{r}}\left|A_{\mathrm{P}}(z, t)\right|^{2}+3 / 4 \chi_{\mathrm{pm}} \times\right.\right. \\
\left.\left.\left.\times\left(\left|A_{\mathrm{R}}(z, t)\right|^{2}+2\left|A_{\mathrm{P}}(z, t)\right|^{2}\right)\right] \times A_{\mathrm{R}}(z, t) \exp \left[i\left(\beta_{\mathrm{R}} z-\omega_{\mathrm{R}} t\right)\right]\right]+ \text { c.c. }\right\}
\end{aligned}
$$

where $\chi_{\mathrm{r}}$ and $\chi_{\mathrm{pm}}$ are the imaginary and real parts of $\chi^{(3)}$.

The nonlinear polarization at the pump (or Raman) frequency depends not only on the peak power, but also on the Raman (or pump) peak power. This is an important fact which should have general implications in processes where several pulses propagate simultaneously in the same or opposite direction (stimulated Raman scattering, stimulated four photon mixing, cross talk, soliton interaction [79], frequency multiplexing [80, 81], temporal modulation instabilities, [82, etc.]) at different frequencies.

Using the expressions of $P_{\mathrm{P}}^{(\mathrm{NL})}$ and $P_{\mathrm{R}}^{(\mathrm{NL})}$ in the nonlinear wave equation, Schadt et al. derived the nonlinear and dispersive equations for Raman and pump pulses [76-77]. In the first-order approximation, assuming small Raman conversion, neglecting losses and group velocity dispersion but keeping the pulse walk-off, the pump envelope $A_{\mathrm{P}}=a \mathrm{e}^{i \alpha}$ and the Raman envelope $A_{\mathrm{R}}=b \mathrm{e}^{i \beta}$ are described by the set of differential equations :

$$
\begin{gathered}
\partial a / \partial z+1 / v_{\mathrm{P}} \partial a / \partial t=0 \\
\partial \alpha / \partial z+1 / v_{\mathrm{P}} \partial \alpha / \partial t=1 / 2 \gamma_{\mathrm{pm}} a^{2} \\
\partial b / \partial z+1 / v_{\mathrm{R}} \partial b / \partial t=1 / 2 \delta_{\mathrm{r}} a^{2} b \\
\partial \beta / \partial z+1 / v_{\mathrm{R}} \partial \beta / \partial t=\gamma_{\mathrm{pm}} a^{2}
\end{gathered}
$$

where $v_{i}$ are the group velocities, $\delta_{r}=$ $3 \omega_{\mathrm{P}}^{2} \mu_{0} \chi_{\mathrm{R}} / 4 \beta_{\mathrm{P}}$ is the Raman gain, $\gamma_{\mathrm{pm}}=$ $3 \omega_{\mathrm{P}}^{2} \mu_{0} \chi_{\mathrm{PM}} / 8 \beta_{\mathrm{P}}$ is the phase modulation coefficient, and the wavelength dependences of $\delta_{\mathrm{r}}$ and $\gamma_{\mathrm{pm}}$ have been neglected.

The analytical solutions of equations (20) are given by [78]

$$
\begin{gathered}
a(z, t)=a_{0} F\left[\left(t-z / v_{\mathrm{P}}\right) / \tau_{0}\right] \\
\alpha(z, t)=1 / 2 \gamma_{\mathrm{pm}} a_{0}^{2} F^{2}\left[\left(t-z / v_{\mathrm{P}}\right) / \tau_{0}\right] z \\
b(z, t)=b_{0} G\left[\left(t-z / v_{\mathrm{R}}\right) / \tau_{0}\right] \times \\
\times \exp \left[1 / 2 \delta_{\mathrm{r}} a_{0}^{2} \psi(z, t)\right]
\end{gathered}
$$

$$
\begin{gathered}
\beta(z, t)=\gamma_{\mathrm{pm}} a_{0}^{2} \psi(z, t) \\
\psi(z, t)=\int_{0}^{z} F^{2}\left\{\left[t-z / v_{\mathrm{R}}-\right.\right. \\
\left.z^{\prime}\left(1 / v_{\mathrm{P}}-1 / v_{\mathrm{R}}\right) / \tau_{0}\right\} \mathrm{d} z^{\prime}
\end{gathered}
$$

where $F$ and $G$ are the initial pump and Raman pulse shapes; and $\psi(z, t)$ is the pump-Raman overlap function which plays a significant role on the temporal and spectral shapings of the Raman pulse.

The XPM-affected spectral distribution of the Raman pulse is readily obtained by computing the Fourier transform of the complex envelope $A_{\mathrm{R}}(z, t)$.

As shown by equation (21d), the Raman pulse yields a cross phase modulation $\beta(z, t)$ generated by the co-propagating pump pulse. This dephasing is linearly proportional to the pump intensity and the overlap function $\psi(z, t)$. The time derivative of $\beta(z, t)$ leads to frequency shifts and spectral broadening as in the SPM case. The pulse walk-off is important for its introduces an asymmetry in the cross phase modulation (assuming the Raman pulse has an initial pulse shape $G(\tau)$ ). The Raman pulse goes faster than the pump pulse in the normal dispersion regime. The cross phase peak lags behind the Raman peak and the pulse sees mainly the dephasing corresponding to red-shifted frequencies originating from the laser pulse front. Consequently, 1) the Raman peak is shifted toward the Stokes frequencies, and 2) the spectral broadening is larger for Stokes frequencies.

In the dispersionless approximation, the Raman/pump broadening ratio can be estimated by [35],

$$
\Delta \omega_{\mathrm{R}} / \Delta \omega_{\mathrm{P}}=\left\{4\left[1+\left(\delta_{\mathrm{r}} / 2 \gamma_{\mathrm{pm}}\right)^{2}\right]\right\}^{0.5} .
$$

Notice that for silica $\delta_{\mathrm{r}} / 2 \gamma_{\mathrm{pm}} \ll 1$ and $\Delta \omega_{\mathrm{R}} / \Delta \omega_{\mathrm{P}}$ $\approx 2$.

As shown by equation (21c), the temporal shape of the Raman pulse is also strongly affected by the pulse walk-off occurring during the gain process. The shape is distorted. The leading edge becomes 
smoother and the trailing edge sharper. However, at this order of approximation, XPM has not effect on the pulse shape.

\subsection{MEASUREMENTS OF CROSS PHASE MODU- LATION.}

3.2.1 XPM of picosecond pulses in optical fibers [3637]. - Emission spectra of 25-ps pulses propagating in a 10-m long singlemode optical fiber are shown in figure 12 as a function of the pump pulse energy. The spectrum of weak pump pulses is shown in figure 12a. The narrow spectral width is resolutionlimited at $0.2 \mathrm{~nm}$. For higher input energies, SPM, SRS and XPM affect the pulse propagation, and significantly change the spectra. Figure $12 \mathrm{~b}$ shows the spectral intensity distributions of the pump and first Raman lines. The Raman shift is about $440 \mathrm{~cm}^{-1}$ which is characteristic of SRS shifts in silica. As predicted, the Raman line, which is broadened by SPM and XPM, is wider than the laser line. For increasing pump pulse energy the Raman

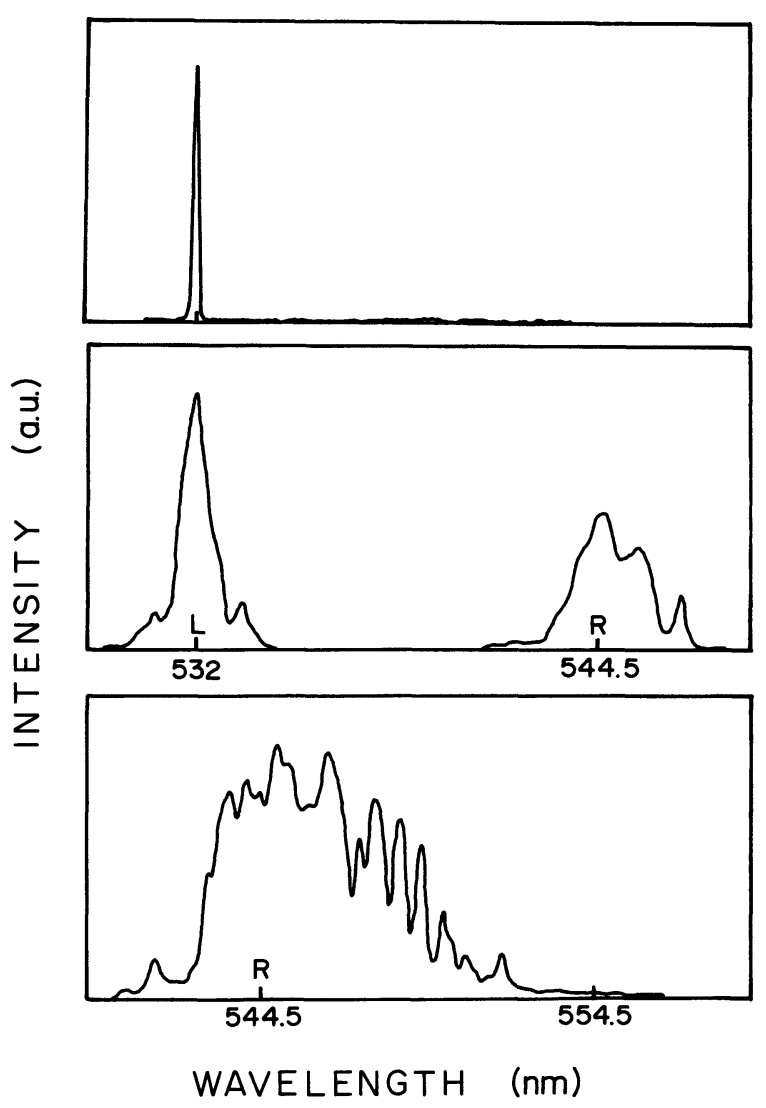

Fig. 12. - Cross phase modulation (XPM) and self phase modulation (SPM) effects generated by $25 \mathrm{ps}$ pulses in optical fibers. (a) Laser line. (b) SPM-broadened laser line and XPM-broadened Raman line at $440 \mathrm{~cm}^{-1}$. (c) Wide broadening of the Raman line by XPM and SPM. line broadens more (Fig. 12c), and clearly displays spectral features of XPM and SPM :

- wide spectral width,

- fast spectral modulation characteristic of phase modulation broadenings,

- Stokes broadening of Raman lines larger than the anti-Stokes broadening, and

- slight spectral shift of the maximum intensity peak toward the Stokes frequencies.

3.2.2 XPM of femtosecond pulses in ethanol. Ethanol has a Raman line shifted by $2928 \mathrm{~cm}^{-1}$. Figures 13 and 14 show spectra of Stokes and antiStokes Raman lines as a function of pump intensity. These spectra were obtained using $1 \mathrm{~mJ}-500$ fs pulses slightly focused into a $20 \mathrm{~cm}$-long cell filled with ethanol. A low-intensity Stokes Raman spectrum is shown in figure 13. For higher input pump energies, Stokes Raman spectra were broadened by SPM and XPM. Spectral shapes were similar to those measured for picosecond Raman pulses generated in optical fibers (Fig. 12). In particular, the spectra were asymmetrically broaden towards the longest wavelengths which is characteristic of the pulse walk-off.

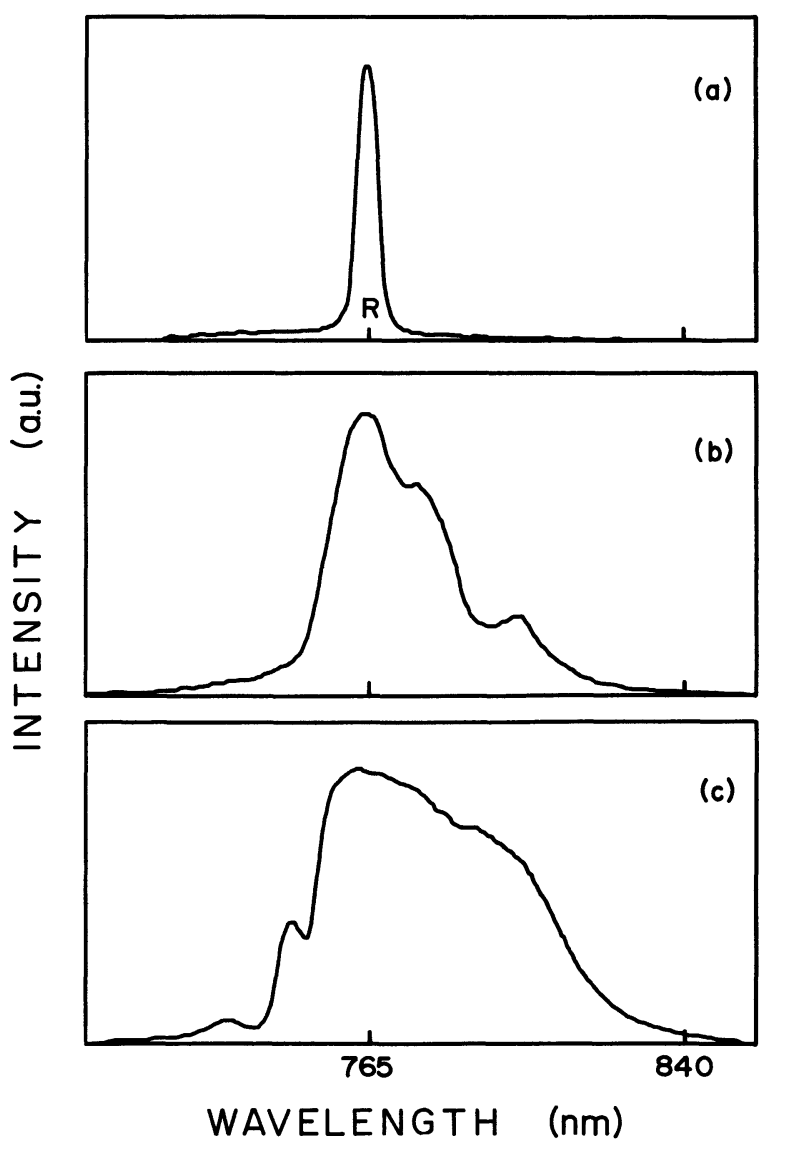

Fig. 13. - Cross phase modulation (XPM) and self phase modulation (SPM) effects generated by 500 -fs pulses on the Stokes Raman of ethanol. 


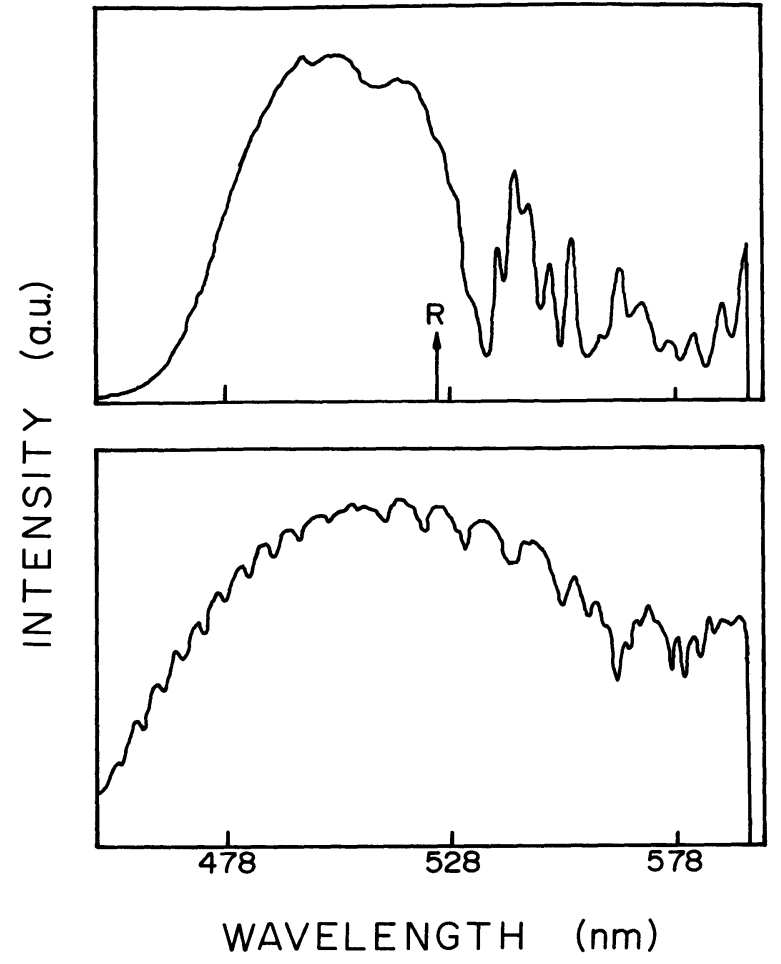

Fig. 14. - Cross phase modulation (XPM) and self phase modulation (SPM) effects generated by 500 -fs pulses on the anti-Stokes Raman of ethanol.

Typical spectra of the anti-Stokes Raman line are displayed in figure 14. They were as wide as Stokes spectra but with tails pointing towards the shortest wavelengths. This asymmetry can also be explained by the XPM theory if we assume the Raman pulse has an initial shape. Anti-Stokes Raman pulses propagate slower than pump pulses. As a consequence of the walk-off, the Raman pulse sees mainly the nonlinear phase produced by the pump-pulse trailing edge. More blue-shifted frequencies are generated about the anti-Stokes Raman line and the broadening is enhanced toward the shortest wavelengths. These are signatures of walk-off. One should notice that modulations on the right side of the spectra are coming from the SPM pump broadening.

\section{Induced phase modulation (IPM).}

Induced phase modulation (IPM) is comparable to self phase modulation. IPM occurs when the intense electric field of a pump pulse is used to modulate the refractive index of a co-propagating weak pulse of a different carrier frequency which is not generated in the medium by the pump pulse. The fact that probe and pump pulses are generated independently and outside of the interactive medium allows a total control of the probe phase modulation which makes IPM qualitatively different from XPM. Hence, the spectral and temporal properties of probe pulses can be controlled by intense pump pulses, which can lead to important applications such as optical computing and frequency coding. Enhancement of spectral broadening using IPM was first predicted and experimentally observed by our group in 1985-1986 [33-34]. Strong picosecond pulses generated by a ND : glass laser at $1054 \mathrm{~nm}$ were successfully used to increase the phase modulation and widen the continuum produced by $527-\mathrm{nm}$ weaker pulses which propagated in a BK-7 glass. Recently, we observed IPM effects in optical fibers using 25-ps pulses.

In this section, experimental measurements of IPM in BK-7 glasses are reviewed and new results of IPM using optical fibers are reported.

4.1 Measurements of induced phase moduLATION IN BULK GLASSES [34]. - A mode-locked $\mathrm{Nd}$ : glass laser was used to generate 8-ps pump pulses at $1054 \mathrm{~nm}$ and weak second-harmonic pulses at $527 \mathrm{~nm}$. The energy of the $527-\mathrm{nm}$ pulses was kept nearly constant at about $80 \mu \mathrm{J}$, while the energy of the $1054-\mathrm{nm}$ pump pulses was varied from 0 to $2 \mathrm{~mJ}$. Both pulses entered simultaneously into a 9-cm-long BK-7 glass after having been weakly focused.

Experimental results are shown in figure 15. A weak supercontinuum signal was measured when only the $527-\mathrm{nm}$ pulse propagated into the sample. An enhanced supercontinuum signal was observed when both pulses were sent through the sample. The average energy of the supercontinuum spectrum contained in the band $410-660 \mathrm{~nm}$ was calculated, by spectral integration, to be 11 times larger in the presence of $1054-\mathrm{nm}$ pump pulses than with the 527$\mathrm{nm}$ probe pulses only.

Since the supercontinuum generation of picosecond pulses can arise either from phase modulation or from four photon parametric generation

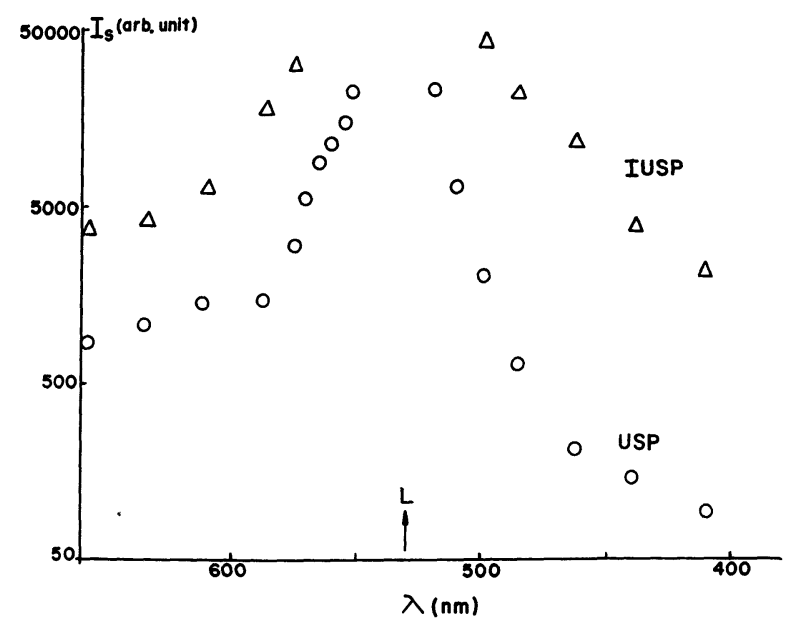

Fig. 15. - Spectrum of an induced ultrafast supercontinuum pulses (IUSP) generated by induced phase modulation (IPM), and spectrum of an supercontinuum pulse (USP) generated by self phase modulation [34]. 
(FFPM), it is important to differentiate the respective contributions. In bulk materials, four photon parametric frequencies are generated in angular wing patterns whose angles are determined by the phase matching conditions. SPM frequencies are generated collinearly to the beam propagation. Therefore, spatial filtering can be used to separate and measure each contribution. In the inducedcontinuum experiment, we found the FFPM contribution to the spectral energy to be less than $1 \%$. Thus, the observed continuum enhancement was attributed to the induced phase modulation mechanism which satisfied the collinear geometry.

4.2 MEASUREMENTS OF INDUCED PHASE MODULATION IN OPTICAL FIBERS. - In a most recent experiment, the IPM spectral effects were measured in an optical fiber. Pump and probe pulses of $30 \mathrm{ps}$ duration were generated at $1064 \mathrm{~nm}$ and at $532 \mathrm{~nm}$ using a Nd : YAG laser and second harmonic crystal. The probe and pump pulses entered the optical fiber either separately or together. Spectra of 532-nm pulses were measured at the fiber output using an optical multichannel analyser OMA 2. The optical

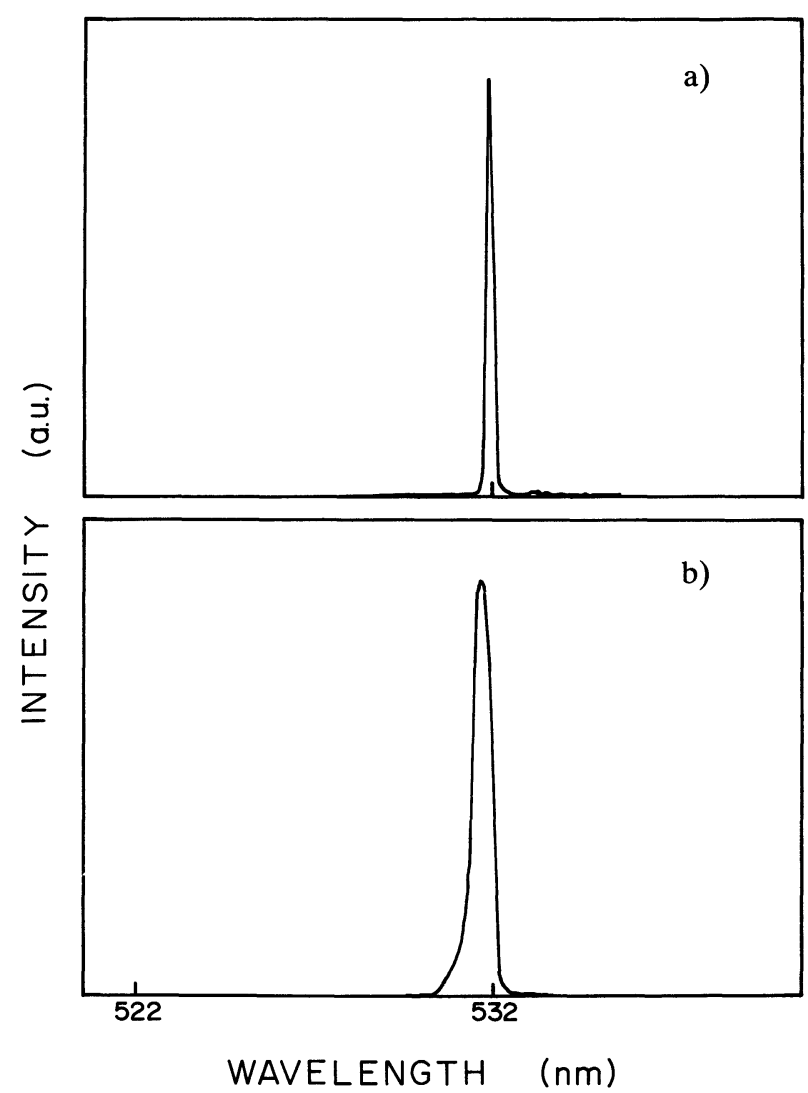

Fig. 16. - Effects of induced phase modulation (IPM) on 25-ps 532-nm pulses in an optical fiber. (a) 532-nm reference laser line. (b) Spectrum of 532-nm pulses having propagated with strong command pulses. The IPM-affected-spectrum is wider, asymmetrical and its peak of maximum intensity has shifted towards the anti-Stokes frequencies. fiber was made by Corning Glass to be singlemode in the $1.3 \mu \mathrm{m}$ region of the spectrum; its length was $20 \mathrm{~m}$.

In figure 16 we have plotted the spectra of $532-\mathrm{nm}$ pulses measured for the two cases. In figure 16a the spectrum of probe pulses having propagated alone in the fiber peaks at $532 \mathrm{~nm}$ and is fairly symmetrical in shape. The spectrum of $532-\mathrm{nm}$ pulses having copropagated with 100 times more intense $1064-\mathrm{nm}$ pulses is shown in figure $16 \mathrm{~b}$. In this latter case, the 532-nm spectrum profile became about three times broader, and had its peak shifted towards the antiStokes frequencies by about $0.4 \mathrm{~nm}$. The shape was also distorted towards the shortest wavelengths. The spectral broadening and peak shift of probe $532 \mathrm{~nm}$ pulses are plotted, in figures 17 and 18, versus increasing $1064 \mathrm{~nm}$ pump intensities, respectively.

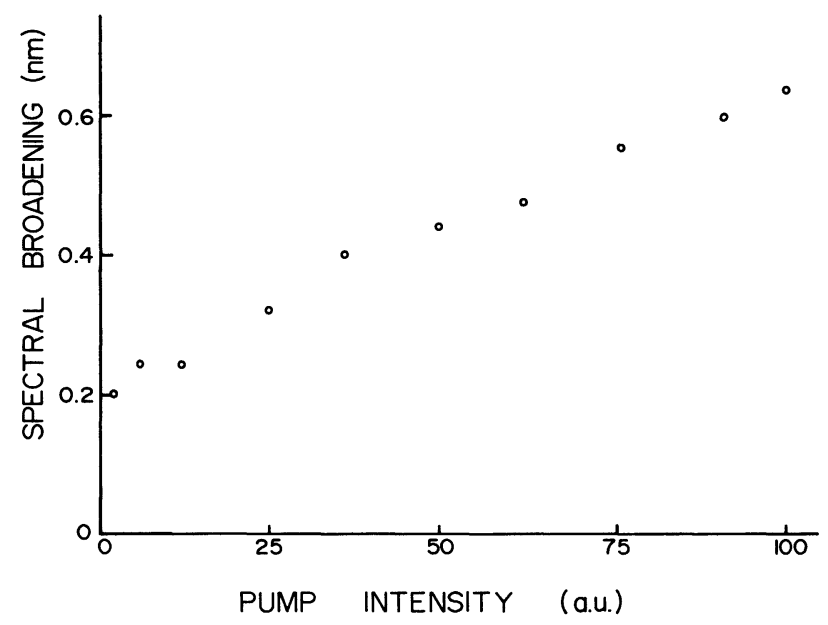

Fig. 17. - Induced spectral broadening of 532-nm pulses versus the intensity of the $1064-\mathrm{nm}$ control pulse.

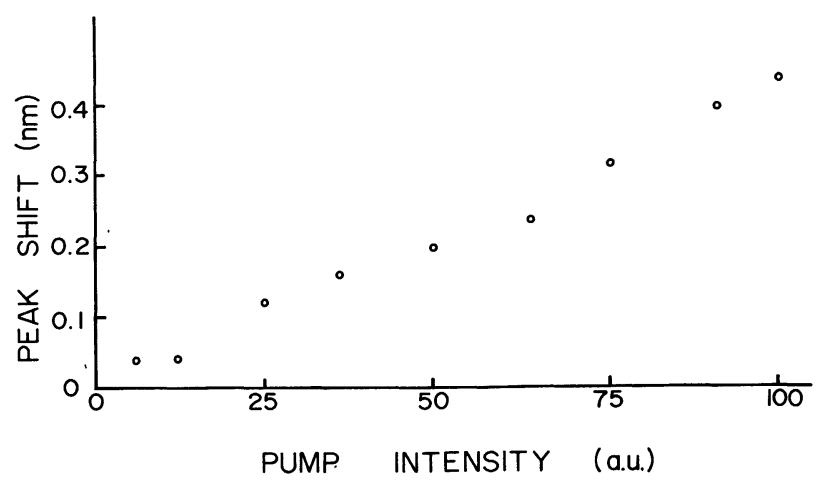

Fig. 18. - Anti-Stokes Peak shifts of 532-nm probe pulses versus the intensity of $1064-\mathrm{nm}$ control pulses.

The IPM signatures are comparable to the XPM features described in section 3. This is not surprising since IPM and XPM are similar phenomena. However, in the XPM experiment, the Stokes Raman peak was shifted towards the Stokes frequencies and 
the broadening was enhanced for Stokes frequencies. The difference is understandable since these asymmetrical features arise from the pulse walk-off. In the XPM experiment the 532-nm pump pulse propagated slower than the 542.5-nm Raman pulse which resulted in a Stokes spectral shift. However, in the IPM experiment the $1064-n m$ pump pulse propagated faster than the 532-nm probe pulse which resulted in an anti-Stokes spectral shift.

The shift of IPM spectral profiles can be explained as shown in figures 19 . The pump pulse $(1064 \mathrm{~nm})$ induces a nonlinear phase at the probe frequency of $532 \mathrm{~nm}$. Due to the group velocity mismatch, the point of maximum phase is generated ahead of the probe pulse peak (Fig. 19). Consequently, the probe pulse sees only the trailing part of the phase induced by pump pulses, which is quasi-linear and can be approximated by

$$
\Delta \phi(\tau)=\phi_{0}-\delta \omega_{0} \tau
$$

where $\delta \omega_{0}$ is the slope of the trailing part of the induced phase and $\phi_{0}$ is an arbitrary pulse. The frequency shift of the entire profile generated by this dephasing is given by

$$
\delta \omega(\tau)=-\partial \Delta \phi(\tau) \partial t=+\delta \omega_{0}
$$

which is the same all over the pulse shape (Fig. 19b). Therefore, the pulse, which had a frequency distribution centred around $\omega_{0}$ in the absence of the pump pulse, has its frequency distribution uniformly shifted by $\delta \omega_{0}$ (anti-Stokes shift) in the presence of strong pulses.

When the probe pulse is strong enough to generate SPM by itself, there is a combined effect of SPM and

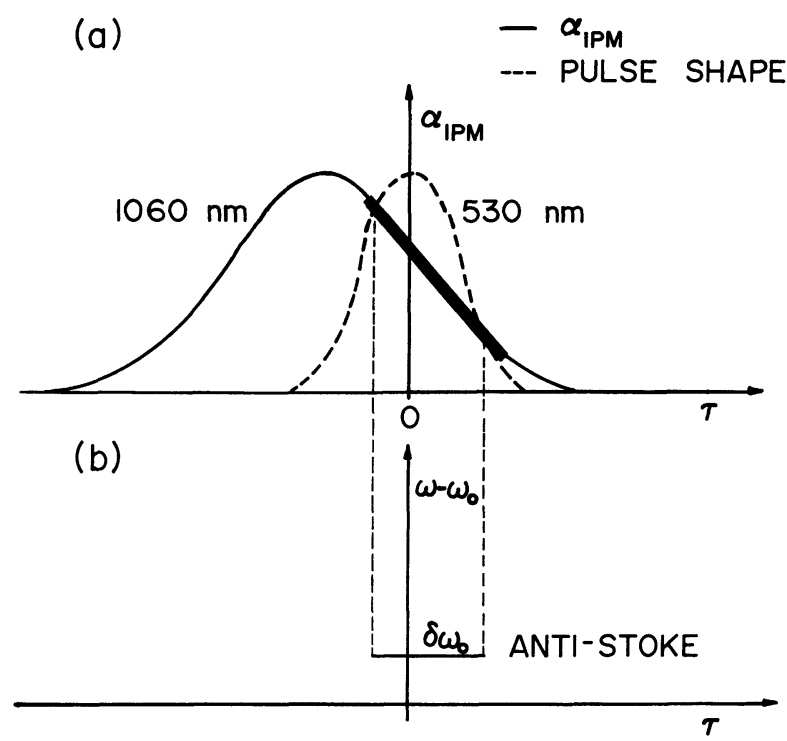

Fig. 19. - Simple model for the combined effect of induced phase modulation (IPM) and pulse walk-off. (a) The induced phase $\alpha_{\text {IPM }}$ is quasi-linear through the probe pulse shape. (b) The frequency shift $\delta \omega=-\partial \alpha / \partial t=$ $\delta \omega_{0}$ is constant through the pulse shapé.
XPM (Fig. 20). The IPM dephasing $\alpha_{\text {IPM }}$ and SPM dephasing $\alpha_{\text {SPM }}$ add. As a result, the probe line simultaneously broadens and shifts. One should notice that in the above IPM experiment using optical fibers, a strong energy conversion from $1064 \mathrm{~nm}$ to $532 \mathrm{~nm}$ occurred from four photon parametric generation. It is likely that combined SPM and XPM effects occurred.

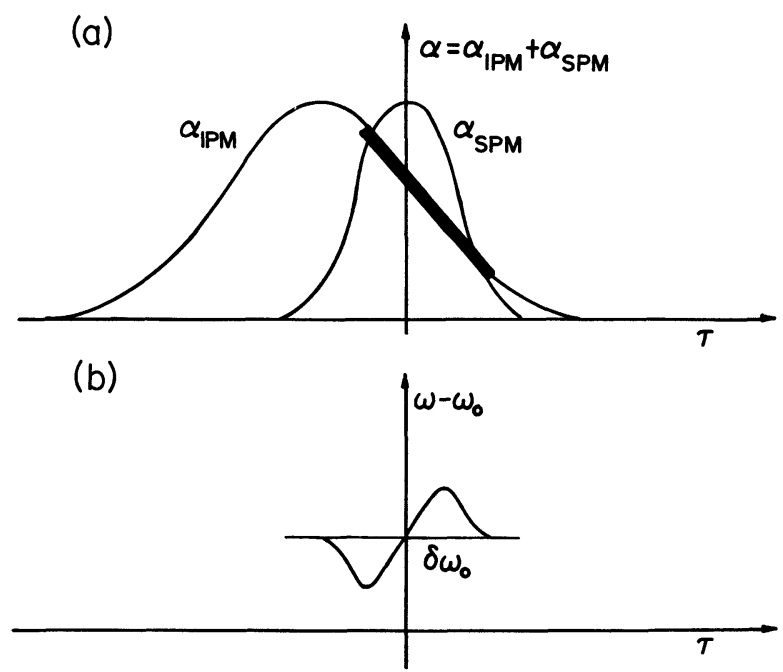

Fig. 20. - Simple model for the combined effects of SPM, IPM and pulse walk-off.

The controllable frequency shift of IPM involves the electronic response of materials. Theoretically, the frequency shift could be turned on and off in a few femtoseconds. Therefore, IPM can be important for optical computing and frequency multiplexing of femtosecond pulses.

\section{Conclusion.}

Supercontinuum generation refers to the generation of bursts of « white » light, which can be obtained by passing intense femtosecond and picosecond pulses through almost any medium. Due to the nonlinear response of the medium, the pulse envelope yields a phase modulation which initiates the wide frequency broadening ( $u$ p to $10000 \mathrm{~cm}^{-1}$ ). The phase modulation of a pulse can be generated either by the pulse itself, a co-propagating pump pulse, or a co-propagating command pulse. These different configurations are called self phase modulation (SPM), cross phase modulation (XPM) and induced phase modulation (IPM), respectively. Both XPM and IPM refer to the phase modulation of a weak pulse by a strong co-propagating pulse of different frequency. However, they are qualitatively different processes. In the XPM scheme, the weak pulse (e.g. the Raman pulse) is generated from the noise, amplified and spectrum broadened by the pump pulse. The pumpprobe frequency difference must satisfy the momen- 
tum conservation law (e.g. the Raman shift frequency of the medium), and there is no external control of the phase modulation. In the IPM case, the weak pulse is generated independently from the pump pulse and outside of the interactive medium. Furthermore, the IPM phase modulation of the weak pulse can be controlled by the experimentator by modifying the command pulse characteristics (peak power, central frequency and time delay). This spectral control is important for it is based on the electronic response of the interaction medium. Consequently, it could be turned on and off in a few femtoseconds which could lead to important applications such as ultrafast optical computing, frequency multiplexing, and tailoring spectra of ultrashort optical pulses.

In this paper, the theoretical and experimental approaches for SPM, XPM and IPM in the process of supercontinuum generation have been described. Moreover, insights have been given on higher order effects arising from group velocity dispersion, selfsteepening, self-focusing, initial pulse chirping, and pulse walk-off. Measurements of SPM have been reviewed, and the newest observations of XPM and IPM effects for femtosecond and picosecond pulses have been presented and their signature described.

\section{Acknowledgments.}

Most of the supercontinuum research using picosecond pulses was initiated by the pioneering work of R. R. Alfano and S. L. Shapiro in the late 1960 's and early 1970's. Recently, the supercontinuum research has been performed in close collaboration with R. R. Alfano, P. P. Ho, T. Jimbo, Z. Li, X. Wang, D. Ji, F. Raccah and P. L. Baldeck. Theoretical studies on self-steepening, induced phase modulation and cross phase modulation were undertaken in collaboration with J. Gersten and J. Manassah. We particularly thank G. Agrawal of AT \& T for theoretical discussions, V. Petricevic and Shu Yang for computer calculations, A. Seas for technical drawing, F. Raccah for technical help with optical fibers, M. R. Junnarkar and Y. Budansky for keeping the CPM laser system operational, N. Ockman for having proofread the manuscript, and R. Dorsinville for his numerous enlightening discussions on nonlinear optics. We thank Corning Glass for the fabrication of the singlemode optical fiber.

From 1985 to 1986, Patrice Baldeck was as a recipient of an exchange program between The City College of New York and the Universite Pierre et Marie Curie (Paris 6 - France) to study at The City College of New York. From 1986 to date, he has been working at the Institute of Ultrafast Spectroscopy and Photonic Application Laboratory, The City College of New York, towards a Ph. D. in electrical engineering on ultrafast laser processes in condensed matter and optical fibers.

This research is supported in part by AFSOR, NSF, PSC/BHE, and Hamamatsu Photonics K. K.
[1] Alfano, R. R., Ultrafast supercontinuum laser source, Proc. Int. Conf. Laser'85, Dec. 2-6 (1985) p. $110-122$.

[2] Alfano, R. R., ed., Biological Events Probed by Ultrafast laser Spectroscopy (Academic Press) 1982 ; Ultrafast Semiconductor Processes, Vol. 1 and 2 (Academic Press) 1984.

[3] Manassah, J., Ho, P., Katz, A. and Alfano, R., Ultrafast supercontinuum laser source, Photonics Spectra Nov. (1984) 53-59.

[4] Ultrashort Light Pulses, 2nd ed., Ed. S. Shapiro (Springer Verlag) 1984.

[5] Alfano, R. R. and Shapiro, S., «Picosecond Spectroscopy using the Inverse Raman Effect », Chem. Phys. Lett. 8 (1972) 631-633.

[6] ShANK, C. V., Measurement of ultrafast phenomena in the femtosecond time domain, Science 219 (1983) 1027-1031.

[7] Dorsinville, D., Delfyett, P. and Alfano, R. R., Picosecond Raman induced phase conjugation in liquids and solids, to be published in Appl. Opt. 26 (1987) 3655-3658.
[8] Knox, W., Fork, R., Downer, M., Solen, R. and SHANK, C., Optical pulse compression to 8-fs at $5-\mathrm{kHz}$ repetition rate, Appl. Phys. Lett. 46 (1985) 1120-1121.

[9] Palfrey, S. L. and Grischkowsky, D., Generation of 16-fs frequency-tunable pulses by optical pulse compression, Opt. Lett. 10 (1985) 562-564.

[10] Alfano, R. R. and Shapiro, S. L., Emission in the region 4 000-7 $000 \AA$ via four-photon coupling in glasses ; Observation of self-phase modulation and small scale filaments in crystals and glasses ; Direct distortion of electronic clouds of rare-gas atoms in intense electric fields, Phys. Rev. Lett. 24 (1970) 584-587, 592-594, 1219-1222.

[11] Bloembergen, N. and Lallemand, P., Complex intensity-dependent index of refraction frequency broadening of stimulated Raman lines and stimulated Rayleigh scattering, Phys. Rev. Lett. 16 (1966) 81-84.

[12] BREWER, R. G., Frequency shifts in self-focused lights, Phys. Rev. Lett. 19 (1967) 8-10. 
[13] Shimizu, F., Frequency broadening in liquids by a short light pulse, Phys. Rev. Lett. 19 (1967) 1097-1100.

[14] Gustafson, T. K., Taran, J. P., Haus, H. A., Lifsitz, J. R. and Kelley, P. L., Self-modulation, self-steepening, and spectral development of light in small-scale trapped filaments, Phys. Rev. 177 (1969) 306-313.

[15] Alfano, R. R., Hope, L. and Shapiro, S., Electronic mechanism for production of self-phase modulation, Phys. Rev. A 6 (1972) 433-438.

[16] Alfano, R. R., Interactions of picosecond pulses with matter, GTE Technical report TR72-330.1 (1972).

[17] Gustafson, T. K., Taran, J. P., Kelley, P. and CHIAO, R., Self modulation of picosecond pulses in electro optic crystals, Opt. Commun. 2 (1970) 17-21.

[18] Kozolskin, V., Malyntin, A., Prokhorov, A., Phase self-modulation of $\mathrm{Nd}$ : glass laser radiation with mode-locking, JEPT Lett. 12 (1970) 150-152.

[19] Ippen, E. P., Shank, C. V. and Gustafson, T. K., Self-phase modulation of picosecond pulses in optical fibers, Appl. Phys. Lett. 33 (1974) 17651767.

[20] Alfano, R., Gersten, J., Zawadzkas, G. and TZOAR, N., Self-phase-modulation near the electronic resonances of a crystal, Phys. Rev. A 10 (1974) 698-708

[21] Yu, W., Alfano, R. R., Sam, C. L. and Seymour, R. J., Spectral broadening of picosecond $1.06 \mathrm{~mm}$ pulse in $\mathrm{KBr}$, Opt. Commun. 14 (1975) 344-347.

[22] Alfano, R., Ho, P., Fleury, P. and GugGENEHEIN, H., Nonlinear optical effects in antiferromagnetic $\mathrm{KNiF}_{3}$, Opt. Commun. 19 (1976) 261-264.

[23] YANG, G. and Shen, Y. R., Spectral broadening of ultrashort pulses in a nonlinear medium, Opt. Lett. 9 (1984) 510-512.

[24] Corkum, P., Ho, P., Alfano, R. and Manassah, J., Generation of infrared supercontinuum covering 3-14 $\mu \mathrm{m}$ in dielectrics and semiconductors, Opt. Lett. 10 (1986) 624-626.

[25] STOlen, R. and LiN, C., Self-phase modulation in silica optical fibers, Phys. Rev. A 17 (1978) 14481453.

[26] Fork, R., Shank, C., Hirliman, C. and Yen, R., Femtosecond white light continua generation, Opt. Lett. 8 (1983) 1-3.

[27] Murav'ev, A. A., Characteristics of generation of a picosecond continuum in a noncentrosymmetric medium, Sov. J. Quantum Electron. 15 (1985) 931-934.

[28] Corkum, P. B., Rolland, C. and Rao, T., Supercontinuum generation in gases, Phys. Rev. Lett. 57 (1986) 2268-71.

[29] Alfano, R., Wanc;, Q., Jimbo, T. and Ho, P., Induced spectral broadening about a second harmonic generated by an intense primary ultrashort laser pulse in ZnSe crystals, Phys. Rev. A 35 (1987) 459-462.
[30] Alfano, R., Wang, Q., Jimbo, T. and Ho, P., Dispersionless propagation of induced spectral broadened pulses in $\mathrm{ZnSe}$, submitted for publication (1987).

[31] Ho, P., LI, Q., Jimbo, T., Ku, Y. and Alfano, R., Supercontinuum generation and propagation in liquid $\mathrm{CCl}_{4}$, Appl. Opt., July 15 (1987).

[32] Ho, P., LI, Q. X., JimbO, T., KU, Y. L. and Alfano, R. R., Supercontinuum pulse generation and propagation in a liquid carbontetrachloride, Appl. Opt. 26 (1987) 2700-2702.

[33] Manassah, J., Musiara, M., Alrano, R. and Ho, $\mathrm{P}$., Induced supercontinuum and steepening of an ultrafast laser pulse, Phys. Lett. 113A (1985) 242-247.

[34] Alfano, R., Li, Q., Jimbo, T., Manassah, J. and Ho, P., Induced spectral broadening of a weak picosecond pulse in glass produced by an intense ps pulse. Opt. Lett. 11 (1986) 626-628.

[35] Gersten, J., Alfano, R. and Belic, M., Combined stimulated Raman scattering and continuum self-phase modulation, Phys. Rev. A 21 (1980) 12221224. Equation (22) has been corrected.

[36] Alfano, R. R., Baldeck, P. L., Raccah, F. and Ho, P., Cross phase modulation measured in optical fibers, Appl. Opt. 26 (1987) 3491-3492.

[37] Baldeck, P. L. and Alfano, R. R., Cross phase modulation effects on spectra of picosecond pulses propagating in singlemode optical fibers, submitted to publication.

[38] Baldeck, P. L. and Alfano, R. R., Intensity effects on the stimulated four photon spectra generated by picosecond pulses in optical fibers, conference abstract \# FQ 7, March meeting of The American Physical Society (1987) ; J. Lightwave Technol. LT-5 (1987).

[39] Bloembergen, N., Nonlinear optics (Benjamin) 1965.

[40] Shen, Y. R., Ed. Principles of nonlinear optics (Academic Press) 1985.

[41] Schubert, M. and Wilhelmi, B., Nonlinear optics and quantum electronics (John Wiley \& Sons) 1986.

[42] Kodama, Y. and Hasegawa, A., Nonlinear pulse propagation in a monomode dielectric guide, IEEE J. Quantum Electron. QE-23 (1987) 510524 and references therein.

[43] Tomlinson, W. J., Stolen, R. H. and Johnson, A. M., Optical wave breaking of pulses in nonlinear optical fibers, Opt. Lett. 9 (1985) 457-459.

[44] Mitschke, F. M. and Mollenauer, L. F., Discovery of the soliton self-frequency shift, Opt. Lett. 11 (1986) 659.

[45] GORDON, J. P., Theory of the soliton self-frequency shift, Opt. Lett. 11 (1986) 662.

[46] Papoulis, A., Systems and transforms with applications in optics (McGraw-Hill) 1968.

[47] Erelyi, A., Asymptotic Expansions (Dover) 1956.

[48] Fisher, R. A. and Bischel, W. K., Numerical studies of the interplay between self phase modulation and dispersion for intense plane wave laser pulses, J. Appl. Phys. 46 (1975) 49214934. 
[49] Pinault, S. C. and Potasek, M. J., Frequency broadening by self-phase modulation in optical fibers, J. Opt. Soc. Am. B 2 (1985) 1318-1319.

[50] Auston, D. H., in Ultrashort Light Pulses, Ed. S. L. Shapiro (Springer-Verlag) 1977.

[51] Masuhara, H., Miyasaka, H., Karen, A. and Mataga, N., TsuchiYa, Y., Temporal characteristics of picosecond continuum as revealed by two-dimensional analysis of streak images, Opt. Commun. 4 (1983) 426.

[52] Li, Q. X., Jimbo, T., Ho, P. P. and Alfano, R. R., Temporal distribution of picosecond super-continuum generated in a liquid measured by a streak camera, Appl. Opt. 25 (1986) 1869-1871.

[53] Dorsinville, R., Delfyett, P. and Alfano, R. R., Generation of 3 ps pulses by spectral selection of the super-continuum generated by a $30 \mathrm{ps}$ second harmonic Nd:YAG laser pulse in a liquid, submitted to Appl. Opt. (1987).

[54] Overviews and references on self focusing in condensed media are given by Auston, D. H., in Ultrafast Light Pulses, Ed S. L. Shapiro (Springer-Verlag) 1977, and by SHEN, Y. R., The Principles of Nonlinear Optics (John Wiley) 1984.

[55] Baldeck, P. L., Raccah, F. and Alfano, R. R., Observation of self focusing in optical fibers with picosecond pulses, Opt. Lett. 12 (1987) 588-589.

[56] Fisher, R. A. and Bishel, W. K., Numerical studies of the interplay between self-phase modulation and dispersion for intense plane-wave laser pulses, J. Appl. Phys. 46 (1975) 4921-4934.

[57] Marcuse, D., Pulse distortion in single-mode optical fibers, Appl. Opt. 19 (1980) 1653-1660.

[58] Potasek, M. J., Agrawal, G. P. and Pinault, S. C., Analytical and numerical study of pulse broadening in nonlinear dispersive optical fibers, J. Opt. Soc. Am. B 3 (1986) 205-211.

[59] Bourkoff, E., ZhaO, W. and JOSEPH, R. I. Evolution of femtosecond pulses in singlemode fibers having higher-order nonlinearity and dispersion, Opt. Lett. 12 (1987) 272-274.

[60] Agrawal, G. P. and PuTasek, M. J., Nonlinear pulse distortion in single-mode optical fibers at the zero-dispersion wavelength, Phys. Rev. A 33 (1986) 1765-1776.

[61] Di. Mari ini, F., Townes, C. H., Gusiafsun, T. K. and Kelley, P. L., Self-steepening of light pulses, Phys. Rev. 164 (1967) 312-322.

[62] ANDERSON, D. and LisAK, M., Nonlinear asymmetric self-phase modulation and self-steepening of pulses in long optical waveguides, Phys. Rev. A 27 (1983) 1393-1398.

[63] YANG, G. and SHEN, Y. R., Spectral broadening of ultrashort pulses in a nonlinear medium, Opt. Lett. 9 (1984) 510-512.

[64] Manassah, J. T., Alfano, R. R. and Mustafa, M., Spectral distribution of an ultrashort supercontinuum laser source, Phys. Lett. A 107 (1985) 305-309.

[65] Manassah, J. T., Mustafa, M. A., Alfano, R. R. and $\mathrm{Ho}, \mathrm{P} . \mathrm{P}$., Spectral extent and pulse shape of supercontinuum, IEEE J. Quantum Electron. QE-22 (1986) 197-204.
[66] Ishida, Y., Naganuma, K., Yagima, T. and Lin, C., Ultrafast self-phase modulation in a colliding pulse mode-locking ring dye laser, in Ultrafast Phenomena 4 (1984) 69-71.

[67] Agrawal, G., private communication (1987).

[68] Cornelius, P. and Harris, L., Role of self-phase modulation in stimulated Raman scattering from more than one mode, Opt. Lett. 6 (1981) 129131.

[69] Dianov, E. M., Karasik, A. Ya., Mamyshev, P. V., Onishchukov, G. I., Prokhorov, A. M., StelMAKH, M. F. and FormicheV, A. A., Picosecond structure of the pump pulse in stimulated Raman scattering in a singlemode optical fiber, JETP Lett. 39 (1984) 691.

[70] LU HIan-HUa, LI YU-LiN and JIANG Jia-LiN, On combined self-phase modulation and stimulated Raman scattering in optical fibers, Opt. Quantum Electron. 17 (1985) 187.

[71] Stolen, R. H. and Johnson, A. M., The effect of pulse walk-off on stimulated Raman scattering in fibers, IEEE J. Quantum Electron. QE-22 (1986) 2154-60.

[72] French, P. M. W., Gomes, A. S. L., GouveiaNeto, A. S. and TAylor, J. R., Picosecond stimulated Raman generation, pump pulse fragmentation, and fragment compression in singlemode optical fibers, IEEE J. Quantum Electron. QE-22 (1986) 2230.

[73] Nakashima, T., Nakazawa, M., Nishi, K. and KubUTA, H., Effect of stimulated Raman scattering on pulse-compression characteristics, Opt. Lett. 6 (1987) 404-406.

[74] Johnson, A. M., Stolen, R. H. and Simpson, W. M., The observation of chirped stimulated Raman scattered light in fibers, in Ultrafast Phenomena 5 (Springer Verlag) 1986.

[75] Islam, M. N., Mollenauer, L. F. and Stolen, R. H., Fiber Raman amplification soliton laser, in Ultrafast Phenomena 5, Eds G. R. Fleming and A. E. Siegman, vol. 46 of Springer Series in Chemical Physics (Springer-Verlag, Berlin) 1986, p. 47.

[76] SChadi, D., Jaskorzynska, B. and OsterberG, U., Numerical study on combined stimulated Raman scattering and self-phase modulation in optical fibers influenced by walk-off between pump and Stokes pulses, J. Opt. Soc. Amer. B 3 (1986) 1257-1260.

[77] SChadT, D. and JaskorzynSKa, B., Frequency chirp and spectra due to self-phase modulation and stimulated Raman scattering influenced by pulse walk-off in optical fibers, J. Opt. Soc. Am. B 4 (1987) 856-862.

[78] Manassah, J. T., Induced phase modulation of the Raman pulse in optical fibers, Appl. Opt. 26 (1987) 3747-3749.

[79] Mirschke, F. M. and Mollenauer, L. F., Experimental observation of interaction forces between solitons in optical fibers, Opt. Lett. 5 (1987) 355-357. 
[80] Chraplyvy, A. R. and Stone, J., Measurement of cross phase modulation in coherent wavelengthdivision multiplexing using injection lasers, Elect. Lett. 20 (1984) 996-997.

[81] Chraplyvy, A. R., Marcuse, D. and Henry, P. S., Carrier-induced phase noise in angle-modulated optical-fiber systems, J. Lightwave Technol. LT-2 (1984) 6-10.
[82] Agrawal, G. P., Modulation instability induced by cross-phase modulation, Phys. Rev. Lett. 59 (1987) 880-883.

[83] Shimizu, F. and STOICHEFF, B. P., IEEE J. Quantum Electron. QE-5 (1969) 544.

[84] Duguay, M. A. and Hansen, J., Appl. Phys. Lett. 15 (1969) 192. 